\title{
sur une approche intégrée de l'interaction sismique sol-structure*
}

\author{
on a unified approach \\ to linear seismic soil-structure interaction analysis
}

\author{
Denis AUBRY \\ École Centrale de Paris"*, Professeur
}

\section{Résumé}

Grâce à l'utilisation du principe des travaux virtuels, il est possible de présenter une approche unifiée de l'interaction sismique sol-structure mettant en évidence les sollicitations exercées d'un système sur l'autre adaptées à la cinématique de chaque domaine. La considération du problème sismique au niveau de la fondation est avantageuse car celle-ci peut souvent être considérée comme rigide.

Le raisonnement en sous-structures développé ici ne repose que sur l'hypothèse de linéarité du comportement. Cette hypothèse restrictive étant retenue, le problème complet de l'interaction est envisagé en insistant sur une utilisation d'un champ sismique local le plus riche possible. Divers types de fondations sont envisagés. Le traitement numérique n'est développé que le plus tardivement possible de manière à laisser la voie ouverte aux techniques les plus adaptées et à la mise en œuvre de méthodes simplifiées.

Cette approche modulaire permet d'utiliser en tant que de besoin divers outils, en particulier tout code classique de calcul dynamique des structures.

\begin{abstract}
The principle of virtual work provides an integrated tool to the analysis of seismic soil-structure interaction with a rational definition of the interaction forces between the different components by taking into account the kinematic assumptions relative to each subsystem. Using the foundation displacements as the primary dependant variables is interesting for the foundation may often assumed to be rigid.

The substructure approach presented in this paper relies solely on the assumption of linearity of soil behaviour. With this restrictive hypothesis in mind the full interaction problem is tackled with special emphasis on the necessity to incorporate a qualified seismic free field accounting possible local site effects. Several foundation types are discussed. The numerical techniques are considered only when all the equations of the continuous media have been established in order to implement only those well adapted to each subsystem or to let the possibility of constructing simplified analysis.
\end{abstract}

The presented modular approach allows to use when needed already existing tools especially any standard structural dynamics software.

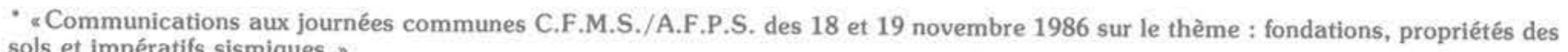
sols et impératifs sismiques.

* 92295 Châtenay Malabry Cedex.
} 


\section{INTRODUCTION}

Lors de ces dix dernières années, de nombreux travaux ont considérablement amélioré l'état des connaissances dans le domaine de l'interaction sismique solstructure (ISSS) à la fois du point de vue des méthodes de calculs, des études expérimentales et de l'évaluation des enregistrements des données sismiques. Dans la pratique, les méthodes ont cependant moins évolué : une représentation du sol par des ressorts et amortisseurs concentrés indépendants de la fréquence ajustés en considérant le sol comme un demi-espace élastique homogène est encore souvent utilisé ainsi que la méthode des éléments finis directe en bidimensionnel pour des sites hétérogènes.

Un certain nombre de résultats ont été mis en évidence sur l'influence d'un substratum très profond, de l'hétérogénéité du sol, des ondes de surface, de la forme et de la profondeur des fondations, ou de leur souplesse. Les deux approches précédentes ne permettent pas aisément de prendre en compte ces divers aspects.

Une autre difficulté importante réside dans la modélisation du comportement du sol. En effet, le sol est un matériau poreux très fortement anélastique, les pores étant très souvent occupés par de l'eau. Des lois de comportement récemment proposées dans la littérature (HUJEUX [33]) reposant sur la théorie de l'élastoplasticité et appliquées aux contraintes effectives permettent actuellement de reproduire les aspects essentiels de ce comportement. Des modèles de milieux poreux généralisant la théorie de Biot sont capables de prendre en compte la propagation des ondes en milieu biphasique (MODARESSI [57]). Cependant l'inconvénient actuel de cette approche est qu'elle reste encore très coûteuse de mise en ceuvre. La détermination des paramètres in situ de modèles complexes pose également des difficultés considérables surtout si l'on veut explorer des domaines de déformations importantes. La mise en ceuvre de tels calculs ne sera pas abordée dans ce travail. Cependant lorsque les non-linéarités sont concentrées à l'interface solfondation sous forme de lois de frottement, une approche complète est encore possible comme nous le verrons à la fin.

Lorsqu'une modélisation linéaire, incluant la viscoélasticité, le comportement hystérétique ou la technique non linéaire équivalente, est acceptable, il est possible de traiter le problème complet de l'interaction solstructure. En utilisant des techniques de sous-structures le problème complet tridimensionnel de l'ISSS peut être abordé à l'heure actuelle à un coût raisonnable. Cette approche permet de développer des outils adaptés à chaque domaine : site géologique, site géotechnique, fondations, structure. Des phénomènes complexes, mentionnés plus haut, tels que : effets locaux de site, ondes de surface, interaction structure-solstructure, fondations flexibles et/ou profondes peuvent être pris en compte. Les développements proposés dans ce travail, en ne faisant que cette seule hypothèse de linéarité tentent d'élaborer une approche intégrée des effets de propagation d'ondes et de vibrations dans un système aussi hétérogène.

\section{MOUVEMENT SISMIQUE EM CHAMP LIBRE ET EFFET LOCAL DE SITE}

En utilisant des techniques développées en sismologie (AKI [1]) il est maintenant possible de produire des accélérogrammes synthétiques significatifs de la source et prenant en compte l'hétérogénéité en profondeur. Des difficultés subsistent à haute fréquence mais certains aspects tels que : effets de source, propagation source-site, directivité, effets locaux de site et corrélation entre les composantes du mouvement sismique peuvent être modélisés d'une manière unifiée, rationnelle et consistante. La prise en compte quantitative complète de la séismicité est encore coûteuse car de nombreuses échelles de grandeur différentes interviennent depuis la source jusqu'au voisinage de la structure.

Avant d'élaborer les outils dont nous aurons besoin dans l'analyse de l'interaction sol-structure, il est important de rappeler les effets de propagation des ondes sismiques car elles représentent la véritable sollicitation sur la structure bien qu'il ne soit pas défini directement en terme conventionnel de forces extérieures. Pour des ondes $\mathrm{SH}$ inclinées incidentes sur une fondation superficielle le mouvement sismique induit horizontal est considérablement filtré avec la fréquence, alors qu'un mouvement de torsion en quart de phase se manifeste à ces fréquences. Le même phénomène se produit pour des ondes $\mathrm{P}, \mathrm{SV}$ inclinées ou de Rayleigh avec cette fois-ci un balancement induit important [41] [42] [51] [87]. Ces résultats peuvent être accentués pour des fondations profondes [73]. Ces observations au niveau de la fondation ne sont pas sans conséquence au sein de la structure : des ondes $\mathrm{SH}$ inclinées réduisent les fonctions de transfert à plus haute fréquence, mais peuvent exciter des modes de torsion des structures [3] [29] [30] [34] [49].

Plusieurs échelles interviennent dans la mise en cuvre de l'interaction sol-structure. La première échelle est celle qui est prise en compte dans les calculs sismiques en champ proche de la source. A ce niveau seules interviennent les grandes irrégularités géologiques verticales et le processus de rupture de la faille. La surface du sol apparaît horizontale et bien sûr la structure n'est pas visible. Le signal sismique calculé à la surface horizontale est le champ libre. Si l'on regarde la surface libre d'un peu plus près, les irrégularités apparaissent au niveau de la géologie de surface telles que celles dues à une vallée sédimentaire, qui définira une deuxième échelle. A cette échelle les ondes du champ sismique libre sont diffractées sur cette structure géologique si bien qu'à la surface libre le mouvement sismique sera perturbé par l'effet local de site pour donner le champ local. Une troisième échelle celle de l'interaction sol-structure intervient enfin lorsqu'en s'approchant encore de la surface libre, la structure apparaît, donnant lieu à une deuxième diffraction dont le résultat sera le champ sismique induit.

\subsection{Le champ sismique local}

La mise en œuvre du problème complet de l'ISSS, en particulier dans le cas de fondations profondes impose 
de connaître les accélérations non seulement en surface mais également en profondeur. En général, l'accélération en profondeur décroît quel que soit le type d'ondes incidentes. La contribution des ondes de Rayleigh est souvent faible et décroît avec la fréquence dans les couches sédimentaires, même si elle est plus importante dans les roches. Des études récentes en sismologie (BARD et al. [11] [12] [13] [14], OHTSUKI et al. [64] [65], TRIFUNAC [71], WONG [88]) permettent à l'heure actuelle de mieux comprendre l'influence de divers paramètres.

BARD définit l'effet de site d'origine structural comme l'ensemble des effets liés à la structure géométrique du sol et du sous-sol : topographie de surface, interfaces entre différentes formations géologiques. Les longueurs d'ondes caractéristiques vont de $10 \mathrm{~m}$ à $10 \mathrm{~km}$. Le premier effet bien connu est lié à la stratification plane et aux résonances qui peuvent se développer. Le deuxième effet est lié aux variations latérales tels que des reliefs topographiques ou des remplissages sédimentaires: une augmentation notable des dégâts au sommet de reliefs avec une amplification considérable surtout des mouvements horizontaux est souvent observée. D'une manière générale, une frontière convexe amplifie les mouvements, le contraire se produisant dans le cas d'une frontière concave : dans une vallée l'amplification se produit surtout au bord. Il convient de noter que les effets peuvent créer des ondes de surface loin de la structure.

Les contrastes de propriétés mécaniques amplifient encore les effets de géométrie. L'angle d'incidence des ondes sur ces structures géologiques joue un rôle important et des mouvements différentiels de torsion, flexion, bascule peuvent être créés. Dans le cas de couverture sédimentaire l'amplification est maximale pour une incidence verticale. Les hétérogénéités latérales créent des modes typiquement bidimensionnels avec des ondes de surface piégées. Les effets de site ont également des répercussions sur la durée du signal sismique en surface.

Dans les développements qui vont suivre le champ sismique dont il sera question sera toujours le champ sismique local incorporant les effets de source et de site. Les échelles géologiques de source et de site sont supposées d'ordres de grandeur supérieurs à l'échelle géotechnique si bien que la source et les irrégularités de site sont supposées rejetées à l'infini par rapport aux dimensions de la structure et de la fondation. Cette hypothèse permet à l'évidence de bien séparer les problèmes mais elle introduit de manière essentielle le caractère non borné du domaine de sol à envisager dans le cadre de l'ISSS.

\section{CAS D'UNE FONDATION RIGIDE}

Il s'agit, en quelque sorte du problème canonique de l'interaction sol-structure. Cette hypothèse de rigidité de la fondation est souvent faite et justifiée dans la pratique comme nous le discuterons plus loin. La schématisation des trois domaines : sol, fondation, structure doit être abordée en tenant compte des cinématiques différentes de ces systèmes.

\subsection{Modélisation des trois domaines}

Dans ce paragraphe, les équations nécessaires à la modélisation de l'interaction entre un sol hétérogène viscoélastique, une fondation et une structure élastique linéaire sont rappelées. Aucune méthode numérique n'est envisagée à ce stade. En tenant compte des hypothèses cinématiques dans chaque domaine il est possible de décomposer le problème global en sousproblèmes de tailles plus petites dont ensuite la solution avec des méthodes adaptées pourra être envisagée [4] [5] [52]. La géométrie du problème est indiquée sur la figure ci-dessous ainsi que les notations qui seront employées par la suite.

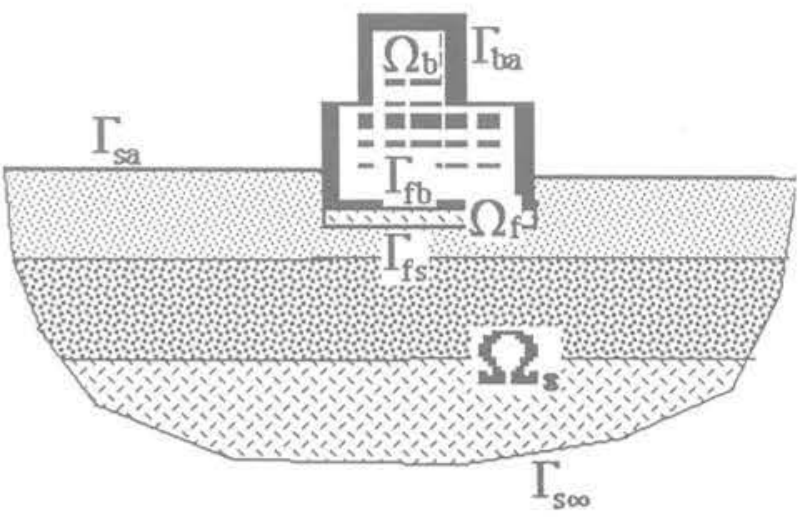

Fig. 1. - Schéma et définition des domaines.

La sollicitation sismique est modélisée par le champ sismique local $u_{i}(x, \omega)$, supposé donné et dépendant de la variable spatiale $\mathrm{x}$ et de la fréquence $\omega$. Nous utiliserons d'une manière générale les notations suivantes pour deux champs scalaires p et q et deux champs vectoriels $u$ et $v$ définis sur un domaine $\Omega_{a}$ :

$$
\begin{aligned}
& (p, q)_{a}=\int \Omega_{\alpha} p(x) q(x) d V \\
& (u, v)_{\alpha}=\int \Omega_{a} u(x) \cdot v(x) d V
\end{aligned}
$$

et pour toute frontière surfacique $\Gamma_{\alpha}$ :

$$
\begin{aligned}
& \langle\mathrm{p}, \mathrm{q}\rangle_{\alpha}=\int_{\mathrm{r}_{\alpha}} \mathrm{p}(\mathrm{x}) \mathrm{q}(\mathrm{x}) \mathrm{dS} \\
& \langle\mathrm{u}, \mathrm{v}\rangle_{\alpha}=\int_{\mathrm{r}_{\alpha}} \mathrm{u}(\mathrm{x}) \cdot \mathrm{v}(\mathrm{x}) \mathrm{dS}
\end{aligned}
$$

Pour deux tenseurs $\mathrm{s}$ et $\mathrm{t}$ le produit contracté suivant est utilisé :

$$
(\mathrm{s}, \mathrm{t})_{\alpha}=\sum_{\mathrm{i}, j} \quad \int \Omega_{\alpha} \mathrm{s}_{i j}(\mathrm{x}) \cdot \mathrm{t}_{i j}(\mathrm{x}) \mathrm{dV}, \quad \mathrm{i}, \mathrm{j}=1,6
$$

On emploiera le double indice $\alpha \beta$ dans la notation ci-dessus à l'interface $\Gamma_{\alpha \beta}$ entre deux domaines $\Omega_{\alpha}$ et $\Omega_{\beta}$. Enfin $\rho_{\alpha}$ désigne la masse volumique du matériau constitutif du domaine $\Omega_{\alpha}$.

\section{2. Élastodynamique du sol}

Le sol occupant le domaine $\Omega_{\mathrm{s}}$ est modélisé par les équations de Navier de l'élastodynamique. 
L'ensemble du problème étant linéaire, il est alors classique de travailler dans le domaine des fréquences. Il suffit que la transformée de Fourier du champ sismique ait été calculée au préalable. Si des variables transitoires sont nécessaires au dimensionnement (efforts maximaux dans la structure par exemple) il conviendra d'effectuer une transformée inverse à la fin. Le champ de déplacement total $u_{s}$ dans le sol doit également vérifier des conditions aux limites :

- de surface libre sur $\Gamma_{s}$,

- internes de continuité de déplacements et de vecteurs contraintes à l'interface entre les couches,

- de continuité de déplacements à l'interface avec la fondation :

$$
\mathrm{u}_{\mathrm{s}}=\mathrm{u}_{\mathrm{f}} \quad, \operatorname{sur} \Gamma_{\mathrm{s} !}
$$

- à l'infini de manière à se raccorder avec le champ sismique de site :

$$
u_{s}=u_{i} \quad, \text { sur } \Gamma_{s \infty}
$$

\subsection{Dynamique de la fondation rigide}

La fondation étant supposée rigide, le champ de déplacements de celle-ci est paramétré par six degrés de liberté $c_{m}$ associés à six fonctions de base $L_{m}$ $(m=1,6)$. Les trois premiers de degré de liberté sont par exemple les trois translations et les trois derniers correspondent à des rotations linéarisées autour du centre de gravité de telle sorte que le déplacement de la fondation s'écrit :

$$
\mathrm{u}_{\mathrm{f}}=\sum_{\mathrm{m}} \mathrm{c}_{\mathrm{m}} \mathrm{L}_{\mathrm{m}} \quad \mathrm{m}=1,6
$$

Afin de décrire la mécanique de systèmes ayant des cinématiques distinctes le principe des travaux virtuels [74] s'avère être l'outil adéquat. En utilisant les notations qui ont été définies au début de ce paragraphe, celui-ci appliqué à la fondation rigide s'écrit :

$$
\begin{aligned}
& -\omega^{2}\left(\rho_{f} u_{f}, v_{f}\right)_{f} \\
& =-\left\langle t_{s}\left(u_{s}\right), v_{f}\right\rangle_{s f}-\left\langle t_{b}\left(u_{b}\right), v_{f}\right\rangle_{b f}
\end{aligned}
$$

pour tout déplacement $v_{f}$ cinématiquement admissible de la fondation. Cette dernière équation est immédiatement équivalente au système suivant :

$$
\begin{aligned}
& -\omega^{2} \sum_{m} c_{m}\left(\rho_{i} L_{m}, L_{n}\right)_{f} \\
& =-\left\langle t_{s}\left(u_{s}\right), L_{n}\right\rangle_{s f}-\left\langle t_{b}\left(u_{b}\right), L_{n}\right\rangle_{b f}, \\
& \mathrm{n}=1,6
\end{aligned}
$$

\section{4. Élastodynamique de la structure}

Soient $u_{b}, \sigma_{b}\left(u_{b}\right), t_{b f}\left(u_{b}\right)$ respectivement le champ de déplacements réels, le champ de contraintes dans la structure et le champ de vecteurs contraintes sur $\Gamma_{b t}$. Soit $v$ un champ de déplacements virtuels cinémati- quement admissibles, c'est-à-dire respectant les conditions d'encastrement de celle-ci sur sa base. Le principe des travaux virtuels appliqué à la structure $\Omega_{b}$ en élastodynamique dans le domaine des fréquences s'écrit :

$$
-\omega^{2}\left(\rho_{b} u_{b}, v\right)_{b}+\left(\sigma\left(u_{b}\right), \varepsilon(v)\right)_{b}=0
$$

A cette étape la structure est considérée comme un solide tridimensionnel. Les conditions aux limites imposées aux déplacements de la structure sont les suivantes :

- conditions de surface libre au contact avec l'air, mais celle-ci est automatiquement prise en compte par le principe des travaux virtuels,

- à l'interface avec la fondation, celle-ci étant supposée rigide, les déplacements de la structure doivent être égaux aux déplacements du sol $u_{1}$ :

$$
\mathrm{u}_{\mathrm{b}}=\mathrm{u}_{\mathrm{f}} \quad \text { sur } \Gamma_{\mathrm{bf}}
$$

Remarque : Dans le cas de plate-forme off-shore, la structure vibre au sein d'un fluide ; il convient de remplacer la condition de surface libre par une condition de raccord avec le fluide. A P'interface entre la structure et le fluide, ce dernier impose la pression :

$$
t_{b f}\left(u_{b}\right)=p n_{t l} \quad \text { sur } \Gamma_{b f l}
$$

où $p$ et $\mathrm{n}_{\mathrm{f}}$ désignent respectivement la pression dynamique dans le fluide et la normale extérieure au domaine correspondant. Il faut ensuite ajouter une condition de continuité du déplacement normal. Les méthodes développées dans (AUBRY, CREPEL [10]) permettent de généraliser l'analyse présentée ici.

\subsection{Décomposition du déplacement du sol}

A ce stade, il est intéressant d'introduire deux décompositions successives du déplacement total du sol, l'une associée à la condition à l'infini, l'autre au mouvement de la fondation. De même dans la structure, il est intéressant de décomposer le déplacement en un déplacement associé au mouvement de la fondation, l'autre à la flexibilité de la structure. Pour se débarrasser de la condition aux limites non homogène sur $\Gamma_{\text {sos }}$, on introduit le champ diffracté total $\mathrm{u}_{\mathrm{d}}$ tel que :

$$
\mathrm{u}_{5}=\mathrm{u}_{\mathrm{i}}+\mathrm{u}_{\mathrm{d}} \quad \text { dans } \Omega_{\mathrm{s}}
$$

La linéarité globale du problème entraîne que toutes les équations et conditions aux limites homogènes que $u_{s}$ et $u_{1}$ vérifient sont également vérifiées par $u_{d}$. Les seules différences se produisent sur $\Gamma_{500}$ et $\Gamma_{s f}$. Par différence, on en déduit immédiatement les conditions imposées à $u_{d}$ à partir de (2) et (3) :

$$
\begin{array}{ll}
u_{d}=0 & \operatorname{sur} \Gamma_{s \infty} \\
u_{d}=-u_{i}+\sum_{m} c_{m} L_{m} & \operatorname{sur} \Gamma_{s i}
\end{array}
$$

La dernière équation suggère une deuxième décomposition du champ de déplacement total $u_{s}$ dans le sol en associant à chaque type de conditions aux limites un champ de déplacement dans le sol. On définit ainsi les 
champs $u_{\text {do }}$ et $u_{d m}(k=1,6)$ solutions de 7 problèmes d'élastodynamique. Chacun de ces champs doit vérifier les équations homogènes de Navier dans $\Omega_{s}$, de surface libre sur $\Gamma_{5}$, de radiation à l'infini sur $\Gamma_{5 \infty}$. Ils diffèrent seulement sur $\Gamma_{\text {si }}$ au contact avec la fondation :

$$
\begin{array}{ll}
u_{d o}=-u_{i} & \text { sur } \Gamma_{s f} \\
u_{d m}=L_{m} & \text { sur } \Gamma_{s f}
\end{array}
$$

si bien que le mouvement total du sol s'écrit :

$$
u_{s}=u_{1}+u_{d o}+\sum_{m} c_{m} u_{d m} \quad \text { dans } \Omega_{s}
$$

En reprenant la définition des champs $\left(u_{1}, u_{d o}, u_{d m}\right)$ on vérifie aisément que $u_{s}$ convient.

\subsection{Décomposition du déplacement de la structure}

A la décomposition du déplacement de la fondation, on associe naturellement la décomposition suivante du déplacement de la structure :

$$
\mathrm{u}_{\mathrm{b}}=\sum \mathrm{c}_{\mathrm{m}} \mathrm{L}_{\mathrm{m}}+\mathrm{u}_{\mathrm{b}}{ }^{*}
$$

si bien que $u_{b}{ }^{*}$ représente le mouvement de celle-ci sur base fixe. Afin de réduire le nombre de degrés de liberté qui interviendront dans le problème couplé, il est agréable de représenter $u_{b}{ }^{*}$ sur la base des modes de vibration de la structure sur base rigide.

Appelons $\Phi^{\mathrm{bA}}$ et $\omega_{A}$ respectivement le mode et la fréquence propres associés. Ce couple vérifie les équations suivantes:

$$
\begin{array}{ll}
\left(\sigma_{b}\left(\phi^{\mathrm{bA}}\right), \varepsilon(v)\right)_{b}=\omega_{A}^{2}\left(\rho_{b} \Phi^{b A}, v\right)_{b} & \\
\Phi^{b A}=0 & \operatorname{sur} \Gamma_{b f} \\
t_{b}\left(\Phi^{b A}\right)=0 & \operatorname{sur} \Gamma_{b a}
\end{array}
$$

pour tout déplacement virtuel de la structure v cinématiquement admissible. En normalisant ces modes propres, on a alors la propriété classique :

$$
\left(\sigma_{b}\left(\Phi^{b A}\right), \varepsilon\left(\Phi^{b B}\right)\right)_{b}=\omega_{A}^{2} \delta_{A B},
$$

où $\delta_{A B}$ désigne le symbole de Kronecker. En décomposant $u_{b}{ }^{*}$ sur les modes $\phi^{b A}$, on peut écrire :

$$
\mathrm{u}_{\mathrm{b}}=\sum_{\mathrm{m}} \mathrm{c}_{\mathrm{m}} \mathrm{L}_{\mathrm{m}}+\sum_{\mathrm{A}} \alpha_{\mathrm{A}} \Phi \mathrm{b} \mathrm{A}
$$

On peut enfin reporter cela dans l'expression des travaux virtuels (6) en tenant compte de (12):

$$
\left(\omega_{B}^{2}-\omega^{2}\right) \alpha_{B}=+\omega^{2} \sum_{m} c_{m}\left(\rho_{b} L_{m}, \phi^{b B}\right)_{b}
$$

Ce qui montre, résultat intuitivement évident, que les amplitudes modales sont fonctions linéaires de chaque degré de liberté de la fondation. En reportant dans (13), on en déduit :

$$
\begin{aligned}
\mathrm{u}_{\mathrm{b}}= & \Sigma_{\mathrm{m}} \mathrm{c}_{\mathrm{m}} \\
& \left\{\mathrm{L}_{\mathrm{m}}+\omega^{2} \Sigma_{\mathrm{A}}\left(\omega_{\mathrm{A}}^{2}-\omega^{2}\right)^{-1}\right. \\
& \left.\left(\rho_{\mathrm{b}} \mathrm{L}_{m}, \Phi^{\mathrm{b} A}\right)_{\mathrm{b}} \Phi^{\mathrm{b} A}\right\}
\end{aligned}
$$

On définit alors la matrice de masse équivalente [52] de la structure $\mathrm{M}_{\mathrm{b}}{ }^{*}$ par :

$$
\begin{aligned}
M_{b}{ }^{*} m= & \sum_{A}\left\{\omega_{A}^{2} /\left(\omega_{A}^{2}-\omega^{2}\right)\right\} \\
& \left(\rho_{b} L_{m}, \phi b A\right)_{b}\left(\rho_{b} L_{n}, \Phi^{b A}\right)_{b}
\end{aligned}
$$

Celle-ci va intervenir dans le paragraphe suivant.

\section{7. Êquation fondamentale de l'ISSS}

Si on reporte alors (12) dans (1), en tenant compte de (11) et de (3), on obtient l'équation suivante en détachant les termes liés au mouvement de la fondation et ceux qui en sont indépendants :

$$
\begin{aligned}
\sum_{m} c_{m}[ & -\omega^{2}\left(\rho_{f} L_{m}, L_{n}\right)_{t} \\
& +\omega^{2}\left\{\sum_{A}\left(\omega_{A}^{2}-\omega^{2}\right)^{-1}\left(\rho_{b} L_{m}, \Phi^{b A}\right)_{b}\right. \\
& \left.<t_{b}\left(\Phi^{b A}\right), L_{n}>_{b i}\right\} \\
+ & \left.<t_{s}\left(u_{d m}\right), L_{n}>_{s t}\right] \\
& =-<t_{s}\left(u_{i}+u_{d o}\right), L_{n}>_{s t} n=1,6
\end{aligned}
$$

Dans l'équation précédente, deux termes ayant une structure identique apparaissent relativement au sol et à la structure, ce sont respectivement :

$$
\begin{aligned}
& \mathrm{K}_{m n}=\left\langle\mathrm{t}_{\mathrm{s}}\left(\mathrm{u}_{\mathrm{dm}}\right), \mathrm{L}_{\mathrm{n}}\right\rangle_{\text {sf }} \\
& \mathrm{B}_{\mathrm{An}}=\left\langle\mathrm{t}_{\mathrm{b}}\left(\Phi^{\mathrm{b} A}\right), \mathrm{L}_{\mathrm{n}}\right\rangle_{\mathrm{bt}}
\end{aligned}
$$

En utilisant la formule de Green dans (18), on obtient :

$$
\begin{aligned}
<\mathrm{t}_{\mathrm{b}} & \left(\Phi^{\mathrm{bA}}\right), \mathrm{L}_{n}>_{\mathrm{bt}} \\
& =\left(\sigma_{\mathrm{b}}\left(\Phi^{\mathrm{bA}}\right), \delta\left(\mathrm{L}_{n}\right)\right)_{b}+\left(\Delta^{*}\left(\Phi^{\mathrm{bA}}\right), \mathrm{L}_{n}\right)_{b} \\
& =-\omega_{A}{ }^{2}\left(\rho_{b} \Phi^{\mathrm{bA}}, \mathrm{L}_{n}\right)_{\mathrm{b}}
\end{aligned}
$$

en tenant compte du fait que $\mathrm{L}_{n}$ est un mouvement de corps rigide et que $\Phi^{\mathrm{bA}}$ est un mode propre de vibration de la structure. Dans (16), on voit alors apparaitre la matrice de masse équivalente de la structure $\mathrm{M}_{\mathrm{b}}{ }^{*}$. En reportant enfin (17) d'une part et (19) et (15) d'autre part dans (16), on obtient le système fondamental de l'ISSS de six équations à six inconnues :

$$
\left[-\omega^{2}\left\{M_{f}+M_{b}^{*}\right\}+K\right] c=F^{*}
$$

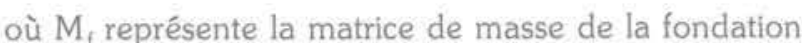
rigide. L'ISSS est totalement contenue dans la matrice $\mathrm{K}$ et le second membre $\mathrm{F}^{*}$ qui représente le système de forces équivalent à la sollicitation sismique.

La matrice $\mathrm{K}$ s'appelle la matrice d'impédance du sol. Le calcul de cette matrice qui nécessite le calcul des champs rayonnés $\mathrm{u}_{\mathrm{dm}}$ et des vecteurs contraintes à 
l'interface avec la fondation résume globalement toute la connaissance apportée par la géotechnique : géométrie et propriétés mécaniques des couches souterraines, géométrie de la fondation [4]. La matrice de masse équivalente de la structure $\mathrm{M}_{\mathrm{b}}{ }^{*}$ doit être fournie par l'ingénieur des structures à partir des modes propres de la structure sur base fixe. Elle dépend de la distribution des masses et de la flexibilité de la structure. Le second membre $\mathrm{F}^{*}$ ne peut être calculé que si le sismologue fournit le champ sismique local $u_{\text {, à l'interface }}$ entre le sol et la fondation donc éventuellement en profondeur si la fondation est enterrée [3].

Si les calculs qui ont mené au système de liSSS (20) ont pu sembler longs, il est remarquable que les coefficients de la matrice d'impédance ont une interprétation physique simple. Il suffit pour cela de revenir aux notations définies au début : si on prend pour $L_{n}$ un terme de translation unitaire correspondant par exemple au vecteur de base $e_{j}$, on obtient:

$$
K_{m n}=\int r_{s f} t_{s}\left(u_{d m}\right) \cdot e, d S=\int r_{s f}\left\{t_{s}\left(u_{d m}\right)\right\} d S
$$

qui fait apparaître la composante numéro j de la résultante des forces développées par le champ de déplacements $u_{d m}$. D'une manière similaire si on prend pour $L_{n}$ un terme de rotation autour de laxe $j$ : $\mathrm{L}_{\mathrm{n}}=\mathrm{e}_{\mathrm{j}} \wedge \mathrm{x}$, on obtient :

$$
K_{m n}=\int_{r s t}\left(t_{s}\left(u_{d m}\right), e_{j}, x\right) d S
$$

qui fait apparaître le moment des mêmes forces autour de cet axe.

La matrice d'impédance du sol est complexe pour deux raisons :

- lorsqu'on passe dans le domaine des fréquences, la viscoélasticité du sol que l'on peut traduire sous forme d'amortissement interne, introduit un terme complexe si bien que les champs $u_{i k}$ sont en général complexes.

- le domaine de sol $\Omega$ s étant non borné, il y a, pour chacun des sept problèmes, transport d'énergie dû à la radiation des ondes à l'infini. Cette perte d'énergie est parfois appelée l'amortissement géométrique. La partie réelle de la matrice d'impédance peut physiquement être assimilée à un système de ressorts concentrés alors que la partie imaginaire à des amortisseurs [31]. Suivant le domaine de fréquences et si la stratification du sous-sol est complexe, la partie réelle peut devenir négative [37] [38] ; il est alors difficile de parler de ressort. Néanmoins on peut alors assimiler la partie réelle à une masse et on pourra parler comme en acoustique d'accession à l'inertie. La profondeur de la fondation augmente sensiblement les parties réelle et complexe de l'impédance. Le couplage entre tamis et balancement est également accentué [15] [27] [28] [29] [47].

Quelques propriétés de la matrice d'impédance peuvent être facilement établies en utilisant la formule de Green en élastodynamique stationnaire, pour un milieu non borné. En effet si $u_{1}$ et $u_{2}$ sont deux solutions des équations de Navier dans $\Omega_{\mathrm{s}}$, vérifiant des conditions aux limites homogènes sur $\Gamma_{s}$, ainsi que des conditions de radiation à l'infini, on a :

$$
\left.<\mathrm{t}_{\mathrm{s}}\left(\mathrm{u}_{1}\right), \mathrm{u}_{2}>_{\mathrm{sf}}-<\mathrm{t}_{\mathrm{s}}\left(\mathrm{u}_{2}\right), \mathrm{u}_{1}\right\rangle_{\mathrm{sf}}=0
$$

La matrice d'impédance est symétrique : il suffit d'appliquer la formule précédente à $u_{\mathrm{dm}}$ et $u_{\mathrm{dn}}$ et de tenir compte de (10). Les termes nuls de $\mathrm{K}$ résultent des symétries du problème appliquées à $u_{\mathrm{dm}}$. En général, il y aura couplage entre l'excitation d'un degré de liberté " $m$ » de la fondation et la force " $n$ » sauf si les conditions de symétrie s'y opposent; il faut alors étudier les symétries de $u_{d m}$.

L'analyse précédente a permis de montrer que le problème de l'ISSS, du point de vue du sol nécessitait la résolution de sept problèmes correspondant respectivement à $u_{\text {do }}$ et $\left(u_{d m}, m=1,6\right)$. Il est possible d'expri-

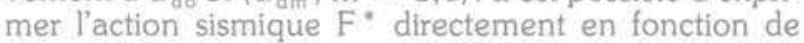
$u_{\mathrm{cm}}$ seulement. En effet, on a toujours en utilisant (21)

$$
\begin{aligned}
\left\langle\mathrm{t}_{\mathrm{s}}\left(\mathrm{u}_{\mathrm{do}}\right), \mathrm{L}_{\mathrm{n}}\right\rangle_{\mathrm{sf}} & =\left\langle\mathrm{t}_{\mathrm{s}}\left(\mathrm{u}_{\mathrm{do}}\right), \mathrm{u}_{\mathrm{dn}}\right\rangle_{\mathrm{sf}} \\
& =\left\langle\mathrm{t}_{\mathrm{s}}\left(\mathrm{u}_{\mathrm{dn}}\right), \mathrm{u}_{\mathrm{do}}\right\rangle_{\mathrm{sf}} \\
& =-\left\langle\mathrm{t}_{\mathrm{s}}\left(\mathrm{u}_{\mathrm{dn}}\right), \mathrm{u}_{1}\right\rangle_{\mathrm{s}}
\end{aligned}
$$

On en déduit que :

$$
\begin{aligned}
F_{n}^{\cdot}= & -<t_{s}\left(u_{1}\right), u_{d n}>_{s f} \\
& +<t_{s}\left(u_{d n}\right), u_{1}>_{s f}
\end{aligned}
$$

Si bien qu'en connaissant uniquement le champ sismique local ainsi que les vecteurs contraintes qu'il développe le long de la fondation, la sollicitation sismique est entièrement déterminée. On notera qu'ici dans (22) la formule de Green n'est pas applicable car le champ sismique $u_{4}$ ne vérifie pas les conditions de radiation.

\subsection{Interaction cinématique et inertielle}

Bien que les développements précédents n'utilisent pas ces termes, il est intéressant de les définir ici car ils sont souvent utilisés dans la littérature. On définit à cet effet le mouvement sismique induit de la fondation caractérisé par le vecteur $c_{\text {, }}$ solution du système linéaire suivant :

(23) $\quad \mathrm{Kc}_{\mathrm{i}}=\mathrm{F}^{*}$

Si la fondation et la structure étaient sans masse, $c_{1}$ serait alors la solution de (20), Dans le cas d'un champ sismique local $u_{i}$ complexe, on ne peut comparer celuicl qui est un champ, avec $c_{1}$ qui est un vecteur de dimension six. Cependant dans la pratique et encore plus dans la réglementation $u$, n'est spécifié qu'en un point dit le point de contrôle. L'interaction cinématique se manifeste alors à ce niveau par la différence éventuelle entre $u_{i}$ en ce point et les trois premières composantes de $c_{1}$. L'intérêt essentiel de $c_{1}$ à ce niveau est seulement qu'il est indépendant de la masse et de la rigidité de la structure.

Une fois le mouvement sismique induit de la fondation déterminé, on peut revenir au mouvement complet de celle-ci en utilisant le système (20). On obtient alors :

$$
\left[-\omega^{2} S\left\{M_{f}+M_{b}^{*}\right\}+I\right] c=c_{1}
$$

où $\mathrm{S}$ est la matrice de complaisance inverse de la matrice $\mathrm{K}$. 


\section{INTERACTION STRUCTURE-SOL-STRUCTURE}

Lorsque les fondations sont distantes approximativement de moins de leur largeur, des effets de couplage apparaissent. Le mouvement de l'une suivant une direction peut déclencher des mouvements de l'autre suivant d'autres directions. Il est tout à fait possible de généraliser la démarche précédente au cas où deux structures sont suffisamment proches pour que les champs diffractés soient modifiés.

L'analyse est différente suivant que les deux structures sont connectées ou non.

Le cas de deux structures indépendantes est tout d'abord envisagé. On indice chaque fondation et chaque structure. Le déplacement de la fondation numéro $\mathrm{M}$, toujours supposée rigide, s'écrit alors :

$$
u_{i M}=\sum_{M m} c_{M m} L_{M m} \quad m=1,6
$$

Il est alors naturel de généraliser la décomposition du champ total dans le sol sous la forme:

$$
u_{s}=u_{i}+u_{d o}+\sum_{M m} c_{M m} u_{d M m} \quad \text { dans } \Omega_{s}
$$

où $u_{\mathrm{do}}$ et les $\mathrm{u}_{\mathrm{dMm}}$ sont caractérisés par les conditions aux limites sur $\Gamma_{\mathrm{fMs}}$ :

$$
\begin{aligned}
u_{d o} & =-u_{i} & & \text { sur } \Gamma_{s f M} \\
u_{d M m} & =L_{M m} & & \text { sur } \Gamma_{s f M} \\
& =0 & & \text { sur } \Gamma_{s f N}
\end{aligned}
$$

Le principe des travaux virtuels appliqué à la fondation $\mathrm{N}$ s'écrit alors :

$$
\begin{aligned}
& \sum_{M m} c_{M m} \\
& \quad\left[-\omega^{2}\left(p_{f} L_{N m}, L_{N n}\right)_{f N} \delta_{M N}\right. \\
& +<t_{b}\left(u_{b N m}\right), L_{N n}>_{b f N} \delta_{M N} \\
& \left.+<t_{s}\left(u_{d M m}\right), L_{N n}>{ }_{s i N}\right] \\
& =-<t_{s}\left(u_{i}+u_{d e}\right), L_{N \pi}>_{s i N} \\
& \quad\left(N=1, N_{f}, n=1,6\right)
\end{aligned}
$$

On voit que dans ce cas les matrices de masse de chaque fondation ainsi que les matrices de masse équivalente des structures ne sont pas couplées. Cependant le couplage structure-sol-structure se manifeste par le fait que la matrice d'impédance généralisée :

$$
\mathrm{K}_{\mathrm{MmNn}}=\left\langle\mathrm{t}_{\mathrm{s}}\left(\mathrm{u}_{\mathrm{dMm}}\right), \mathrm{L}_{\mathrm{Nn}}\right\rangle_{\mathrm{sfN}}
$$

n'est pas bloc-diagonale. Physiquement les colonnes de cette matrice représentent les actions exercées sur la fondation $\mathrm{N}$ par le champ rayonné par la fondation $\mathrm{M}$.

Dans le cas précédent le mouvement de la structure $\mathrm{N}$ ne dépendait que du mouvement de la fondation N. Si les structures sont couplées « par le haut ", il faut introduire une nouvelle décomposition du déplacement $u_{b}$ car un relèvement de corps rigide correspondant au déplacement de la fondation M n'est pas, dans le cas général cinématiquement admissible avec le mouvement de corps rigide de la fondation $\mathrm{N}$ :

$$
\mathrm{u}_{\mathrm{b}}=\sum \mathrm{c}_{\mathrm{Mm}} \mathrm{u}_{\mathrm{bMm}}
$$

où $u_{b M m}$ vérifie les conditions aux limites suivantes sur $\Gamma_{\text {bIM }}$ :

$$
\begin{aligned}
\mathrm{u}_{\mathrm{bMm}} & =L_{\mathrm{Mm}} & & \text { sur } \Gamma_{\mathrm{bfM}} \\
& =0 & & \text { sur } \Gamma_{\mathrm{b} / \mathrm{N}}
\end{aligned}
$$

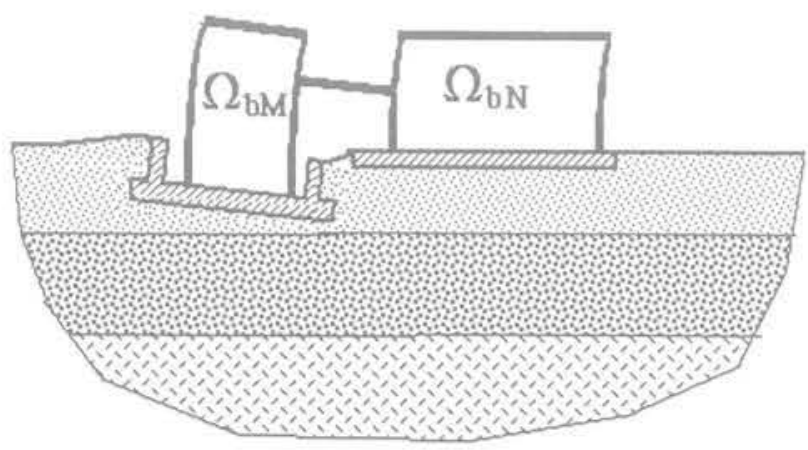

Fig. 2. - Définition du champ $u_{d M m}$

Il n'est plus possible maintenant de définir une matrice de masse équivalente simplement car il n'y a pas de raison d'utiliser comme référentiel de la structure plutôt une fondation que l'autre. Pour chaque degré de liberté $\mathrm{m}$ de chaque fondation $\mathrm{M}$, le code de calculs de structures doit fournir le déplacement $\mathrm{u}_{\mathrm{bMm}}$ et calculer Pimpédance équivalente de la structure au niveau des fondations :

$$
\mathrm{K}_{\mathrm{b}}{ }_{\mathrm{MmNn}}=\left\langle\mathrm{t}_{\mathrm{b}}\left(\mathrm{u}_{\mathrm{bMm}}\right), \mathrm{L}_{\mathrm{Nn}}\right\rangle_{\mathrm{bfN}}
$$

On peut alors généraliser le système de l'ISSS. Il est intéressant de remarquer que le sol et la structure jouent maintenant, vis-à-vis de la fondation un rôle relativement symétrique, les deux matrices d'impé. dance ayant la même structure.

\section{FLEXIBILITÉ DE LA FONDATION SUPERFICIELLE}

Bien qu'en général la fondation ne puisse être considérée comme parfaitement rigide, la présence d'une superstructure relativement rigide a souvent pour effet de renforcer celle-ci si bien que le déplacement de la fondation sera très proche de celui d'un corps rigide. $\mathrm{Si}$ la superstructure est plus souple, la flexibilité de la fondation elle-même doit être prise en compte. A basse fréquence, l'impédance d'une fondation superficielle peut être nettement plus faible que celle d'une fondation rigide. A haute fréquence, au contraire, l'impédance est plus élevée. L'amortissement est par contre toujours plus faible [35] [36] [67]. Si la fondation est relativement massive et doit être modélisée en tant que solide tridimensionnel, elle sera alors avantageusement incorporée dans le maillage de la structure. Il reste alors à étudier le couplage entre une structure étudiée par 
une technique d'éléments finis et un sol dont le champ de déplacements est calculé autrement. Nous aborderons ce problème dans le paragraphe suivant.

Un premier modèle de fondation flexible consiste à la schématiser par un système de plusieurs corps rigides. On est alors ramené au cas de plusieurs fondations avec superstructure connectée envisagée précédemment. Ce modèle est particulièrement intéressant pour des ponts. Si la fondation est mince, on peut alors avancer dans le couplage en tenant compte des hypothèses cinématiques adoptées. On distinguera deux cas suivant qu'il s'agit :

- soit d'un radier souple servant de fondation superficielle ou de paroi moulée servant de fondation profonde ;

- soit de pieux. Ce cas sera examiné dans la section suivante.

Afin de bien mettre en évidence la transition avec le cas précédent nous allons supposer que la fondation $\Omega_{1}$ est constituée de deux parties, une partie rigide $\Omega_{f_{\mathrm{r}}}$ et une

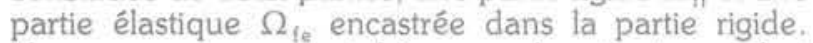
Nous supposons toutefois que la structure n'est connectée qu'à la partie rigide. La figure ci-dessous précise les hypothèses.

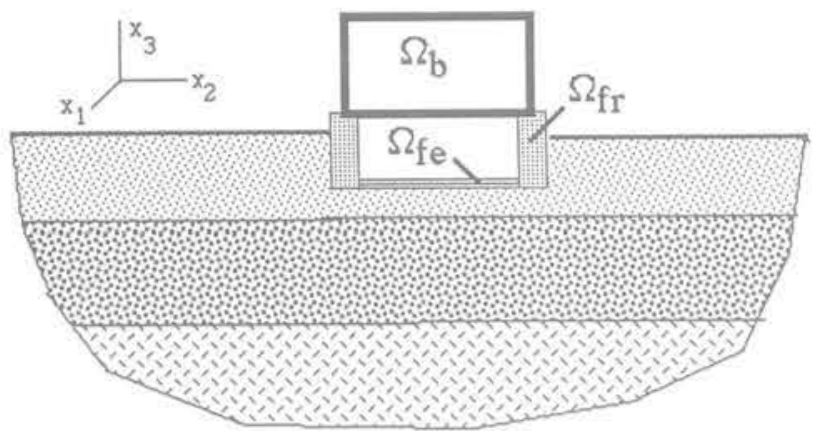

Fig. 3. - Fondation mixte rigidelélastique.

Le déplacement de la partie rigide $\Omega_{\text {ir }}$ s'écrit encore comme précédemment :

$$
\mathrm{u}_{\mathrm{fr}}=\sum_{\mathrm{m}} \mathrm{c}_{\mathrm{m}} \mathrm{L}_{\mathrm{m}} \quad \mathrm{m}=1,6
$$

Alors que, dans le cadre d'une hypothèse cinématique de plaque mince et en négligeant des déformations de membrane de la plaque, le déplacement de la partie flexible $\Omega_{\mathrm{fr}}$ s'écrit :

$$
\begin{array}{r}
u_{i e}=\sum_{m} c_{m} L_{m}-\sum_{\alpha}\left\{x_{3} \partial_{\alpha} u_{f e 3}\right\} e_{\alpha}+u_{f e 3} e_{3} \\
m=1,6 ; \alpha=1,2
\end{array}
$$

où $\mathrm{u}_{\mathrm{fe}_{3}}\left(\mathrm{x}_{\alpha}\right)$ désigne la flèche de la plaque, $e_{\alpha}$ un vecteur unitaire horizontal, $e_{3}$ le vecteur unitaire vertical. L'hypothèse précédente correspond à une cinématique de Kirchhoff-Love, classique en mécanique des structures (WASHIZU [74]). $u_{\text {fe3 }}$ doit vérifier des conditions aux limites d'encastrement sur la frontière $\Gamma_{\text {feft }}$ entre la partie rigide et la partie élastique.

Le principe des travaux virtuels appliqué à la fondation rigide s'écrit :

$$
\begin{aligned}
& -\omega^{2} \sum_{m} c_{m}\left(\rho_{i} L_{m}, L_{n}\right)_{f r} \\
& =- \\
& \quad-<t_{s}\left(u_{s}\right), L_{n}>_{\text {sir }} \\
& \quad-<t_{b}\left(u_{b}\right), L_{n}>_{b f r} \\
& \quad-<t_{i e}\left(u_{i e}\right), L_{n}>_{\text {fefr }} \quad n=1,6
\end{aligned}
$$

Le principe des travaux virtuels appliqué à la fondation flexible s'écrit :

$$
\begin{gathered}
-\omega^{2}\left(\rho_{f} u_{f e}, v_{f e}\right)_{f e}+\left(\sigma_{f e}\left(u_{f e}\right), \varepsilon\left(v_{f e}\right)\right)_{f e} \\
=-\left\langle t_{s}\left(u_{s}\right), v_{i e}\right\rangle_{s f e}
\end{gathered}
$$

en se restreignant à des déplacements $v_{\text {te }}$ cinématiquement admissibles, c'est-à-dire respectant l'encastrement avec $\Omega_{\text {it }}$ ainsi que la cinématique de KirchhoffLove :

$$
v_{f e}=-\sum\left\{x_{3} \partial_{\alpha} v_{f e 3}\right\} e_{\alpha}+v_{f e 3} e_{3} \alpha=1,2
$$

Si on tient compte de (33) et (36) dans (35), cette dernière équation peut s'écrire :

$$
\begin{aligned}
& -\omega^{2} \sum_{m} c_{m}\left(\rho_{i} L_{m}, L_{n}\right)_{f e} \\
& -\omega^{2}\left\{\rho_{f} x_{3}{ }^{2} \partial_{\alpha} u_{f e 3}, \partial_{q} v_{f e 3}\right)_{f e} \\
& \left.+\left(\rho_{f} u_{f e 3}, v_{f e 3}\right)_{f e}\right\} \\
& +\left(\sigma_{f e}\left(u_{f e}\right), \mathcal{E}\left(v_{f e}\right)\right)_{f e} \\
& \left.=+<t_{s a}\left(u_{s}\right), x_{3} \partial_{\alpha} v_{f e 3}\right\rangle_{s f e} \\
& \left.\quad-<t_{s 3}\left(u_{s}\right), v_{f e 3}\right\rangle_{s f e}
\end{aligned}
$$

Grâce à l'hypothèse cinématique, il est toujours possible d'intégrer par rapport à l'épaisseur de la plaque. L'équation précédente devient alors en supposant la plaque symétrique par rapport à sa surface moyenne :

$$
\begin{aligned}
& -\omega^{2} \sum_{m} c_{m}\left(\rho_{f} L_{m}, L_{n}\right)_{f e} \\
& -\omega^{2}\left\{<I_{f} \partial_{\alpha} u_{f e 3}, \partial_{q} v_{f e 3}\right\rangle_{s f e} \\
& \left.\left..+<m_{f} u_{f e 3}, v_{f e 3}\right\rangle_{s f e}\right\} \\
& \left.+<m_{f e}\left(u_{f e 3}\right), k\left(v_{f e}\right)\right\rangle_{s f e} \\
& \left.=<h t_{s a}\left(u_{s}\right), \partial_{a} v_{f e 3}\right\rangle_{s f e} \\
& \left.-<t_{s 3}\left(u_{s}\right), v_{f e 3}\right\rangle_{s f e}
\end{aligned}
$$

où $\mathrm{L}_{\mathrm{f}}, \mathrm{m}_{\mathrm{f}}, \mathrm{m}_{\mathrm{fe}}, \mathrm{k}, \mathrm{h}$ désignent respectivement le moment d'inertie de la plaque, la masse surfacique, le tenseur des moments de flexion et de courbure et la demi-épaisseur de la plaque.

Les termes du membre de gauche de (38) sont classiques du point de vue mécanique des structures. Les termes de droite matérialisent l'interaction sol-structure et mettent en évidence les sollicitations venant du sol adaptées à la cinématique de la plaque. On voit ainsi que le moment des composantes horizontales du vecteur contraint travaille avec la rotation, alors que la contrainte normale travaille avec la flèche.

L'analyse de la structure du problème d'interaction peut encore être affinée en introduisant les modes propres de la plaque dans le vide et encastrée sur la fonda- 
tion. Afin de dégager les termes essentiels sans trop compliquer les équations, nous exprimerons les propriétés de ces modes sur la forme tridimensionnelle du principe des travaux virtuels. L'expression détaillée des divers termes s'obtient évidemment en tenant compte de cette hypothèse. Les modes de vibration de la plaque vérifient une équation identique à ceux de la structure. Nous les rappelons ici : appelons $\phi^{\text {feA }}$ et $\omega_{\text {feA }}$ respectivement le mode et la fréquence propres associés. $\mathrm{Ce}$ couple vérifie les équations suivantes:

$$
\begin{aligned}
& \left(\sigma_{f e}\left(\phi^{f e A}\right), \varepsilon\left(v_{f e}\right)\right)_{f e}=\omega_{f e A}{ }^{2}\left(\rho_{f} \phi^{f e A}, v_{f e}\right)_{f e} \\
& \phi^{f e A}=0 \quad \operatorname{sur} \Gamma_{f e f r}
\end{aligned}
$$

pour tout déplacement virtuel de la plaque $v_{t e}$ cinématiquement admissible. En normalisant ces modes propres, on a alors la propriété classique :

$$
\left(\sigma_{f e}\left(\phi^{f e A}\right), \varepsilon\left(\phi^{f e B}\right)\right)_{f e}=\omega_{f e A}{ }^{2} \delta_{A B}
$$

où $\delta_{A B}$ désigne le symbole de Kronecker. On peut alors écrire en utilisant (33) :

$$
\mathrm{u}_{\mathrm{fe}}=\sum_{\mathrm{m}} \mathrm{c}_{\mathrm{m}} \mathrm{L}_{\mathrm{m}}+\sum_{\mathrm{A}} \alpha_{\mathrm{ieA}} \Phi^{\mathrm{feA}}
$$

On peut enfin reporter cela dans l'expression des travaux virtuels en tenant compte de (14):

$$
\begin{aligned}
& -\omega^{2} \sum_{m} c_{m}\left(\rho_{f e} L_{m}, \phi^{f e B}\right)_{i e} \\
& +\left(\omega_{f e B}^{2}-\omega^{2}\right) \alpha_{f e B}=-\left\langle t_{s}\left(u_{s}\right), \Phi^{i e B}\right\rangle_{s f e}
\end{aligned}
$$

Il est maintenant opportun d'introduire dans le sol divers champs rayonnés qui vont généraliser la décomposition introduite plus haut. On écrit alors :

$$
\begin{array}{r}
u_{s}=u_{1}+u_{d o}+\sum_{m} c_{m} u_{d m}+\sum_{A} \alpha_{f e A} u_{d A} \\
\text { dans } \Omega_{s}
\end{array}
$$

Ces champs sont tous des champs de l'élastodynamique stationnaire vérifiant les équations de Navier dans le sol, les conditions de surface libre sur $\Gamma_{\text {sa }}$ et de radiation sur $\Gamma_{5 \infty}$. Ils diffèrent seulement sur $\Gamma_{\text {sie }} \cup \Gamma_{\text {sir }}$ :

$$
\begin{array}{ll}
u_{d o}=-u_{i} & \text { sur } \Gamma_{\text {sfe }} \cup \Gamma_{\text {sft }} \\
u_{d m}=L_{m} & \text { sur } \Gamma_{\text {sfe }} \cup \Gamma_{s f r} \\
u_{d A}=\Phi^{f e A} & \text { sur } \Gamma_{\text {sfe }} \\
u_{d A}=0 & \text { sur } \Gamma_{\text {sft }}
\end{array}
$$

Physiquement chacun de ces champs correspond soit au mouvement d'ensemble rigide des deux parties, soit à la flexibilité de la fondation élastique alors que la partie rigide est fixe. On peut alors reporter l'expression (43) dans (34) et dans (42).

\subsection{Mouvement de la fondation rigide}

$$
\begin{aligned}
& \sum_{m} c_{m}\left\{-\omega^{2}\left(\rho_{i} L_{m}, L_{n}\right)_{i r}\right. \\
& \left.+<t_{s}\left(u_{d m}\right), L_{n}>_{s i r}\right\} \\
& +\sum_{A} \alpha_{b A}<t_{b}\left(\phi^{b A}\right), L_{n}>_{b f r} \\
& +\sum_{A} \alpha_{f e A}\left\{<t_{s}\left(u_{d A}\right), L_{n}>_{s f r}\right. \\
& \left.+<t_{i e}\left(\phi^{f e A}\right), L_{n}>_{f e f r}\right\} \\
& =-<t_{s}\left(u_{1}+u_{d o}\right), L_{n}>_{s f t}
\end{aligned}
$$

\subsection{Mouvement de la fondation élastique}

$$
\begin{aligned}
& \text { (46) } \left.\quad \sum_{m} c_{m}\right\}-\omega^{2}\left(\rho_{t e} L_{m}, \phi^{f e B}\right)_{t e} \\
& \left.+\left\langle\mathrm{t}_{\mathrm{s}}\left(\mathrm{u}_{\mathrm{dm}}\right), \phi_{\mathrm{fe}} \mathrm{B}\right\rangle_{\mathrm{sfe}}\right\} \\
& +\left(\omega_{\mathrm{feB}}{ }^{2}-\omega^{2}\right) \alpha_{\mathrm{feB}}+\sum_{\mathrm{A}} \alpha_{\mathrm{feA}}<\mathrm{t}_{s}\left(\mathrm{u}_{\mathrm{dA}}\right), \phi^{\mathrm{fe} B}>_{\text {ste }} \\
& =-\left\langle\mathrm{t}_{\mathrm{s}}\left(\mathrm{u}_{1}+\mathrm{u}_{\mathrm{do}}\right), \phi^{\mathrm{fe} B}\right\rangle_{\text {sfe }} \quad \mathrm{B}=1, \mathrm{~N}_{\mathrm{fe}}
\end{aligned}
$$

Il est possible d'obtenir une expression équivalente à (45) en appliquant le principe des travaux virtuels avec un mouvement rigidifiant non seulement à $\Omega_{\text {fr }}$ mais à l'ensemble $\Omega_{\mathrm{fe}} \cup \Omega_{\mathrm{fr}}$. Cela donne en remplacement de (45) une expression plus symétrique et qui fait disparaitre l'interaction entre ces deux parties :

$$
\begin{aligned}
& \sum_{m} c_{m}\left\{-\omega^{2}\left(\rho_{i} L_{m}, L_{n}\right)_{l}\right. \\
& +<t_{s}\left(u_{d m}\right), L_{n}>_{s f\}} \\
& +\sum_{A} \alpha_{b A}<t_{b}\left(\Phi^{b A}\right), L_{n}>_{b f r} \\
& +\sum_{A} \alpha_{f e A}<t_{s}\left(u_{d A}\right), L_{n}>_{s f} \\
& =-<t_{s}\left(u_{1}+u_{d o}\right), L_{n}>_{s f} \quad n=1,6
\end{aligned}
$$

Si on pose successivement :

$$
\begin{aligned}
& K^{\text {te }}{ }_{A n}=\left\langle t_{s}\left(u_{d A}\right), L_{n}\right\rangle_{s f} \\
& K^{e e}{ }_{A B}=\left\langle t_{s}\left(u_{d A}\right), \Phi^{f e B}\right\rangle_{s f e} \\
& K^{e r}{ }_{m B}=\left\langle t_{s}\left(u_{d m}\right), \Phi^{f e B}\right\rangle_{s i e}
\end{aligned}
$$

les équations (46) et (47) sont équivalentes au système suivant, qui généralise (20) :

$$
\begin{aligned}
& {\left[-\omega^{2}\left\{\mathrm{M}_{\mathrm{f}}+\mathrm{M}_{\mathrm{b}}^{*}\right\}+\mathrm{K}\right] \mathrm{c}+\mathrm{K}^{\mathrm{re}} \alpha=\mathrm{F}^{*}} \\
& {\left[-\omega^{2} \mathrm{M}^{e r}+\mathrm{K}^{e x}\right] \mathrm{c}+\left[-\omega^{2} I+\Delta+\mathrm{K}^{e e}\right] \alpha} \\
& =\mathrm{F}^{*}
\end{aligned}
$$

Les autres matrices ont la même signification que dans le cas de la fondation rigide, $\Delta$ est une matrice diagonale de pivot $\omega_{\mathrm{feB}}{ }^{2}$ qui représente la rigidité dynamique de la plaque. Il est important de remarquer que dans (49), $M_{f}$ et $K$ se réfèrent à l'ensemble des deux fonda- 
tions. Par conséquent, dans le cas d'une fondation entièrement rigide, on aurait obtenu $(49)_{1}$, sans le terme $\alpha$, qui apparait donc comme un terme correcteur lorsque cette hypothèse n'est plus satisfaite.

Là encore la formule de Green permet de montrer que les matrices $\mathrm{K}^{\text {te }}$ et $\mathrm{K}^{\text {er }}$ sont transposées l'une de l'autre. En effet, on a :

$$
\begin{aligned}
& \mathrm{K}^{r e}{ }_{A_{n}}=\left\langle\mathrm{t}_{\mathrm{s}}\left(\mathrm{u}_{\mathrm{dA}}\right), \mathrm{L}_{\mathrm{n}}\right\rangle_{\mathrm{si}} \\
& =\left\langle t_{s}\left(u_{d A}\right), u_{d n}\right\rangle_{s i} \\
& =\left\langle t_{s}\left(u_{d n}\right), u_{d A}\right\rangle_{s i} \\
& =\left\langle\mathrm{t}_{\mathrm{s}}\left(\mathrm{u}_{\mathrm{dn}}\right), \phi^{f e A}\right\rangle_{\mathrm{sfe}} \\
& =\mathrm{K}^{e r}{ }_{\mathrm{nA}}
\end{aligned}
$$

\subsection{Remarques sur P'influence de la flexibilité sur l'impédance [23] [35] [36] [67] [75]}

La flexibilité a peu d'effet sur la fréquence fondamentale de mouvement de corps rigide de l'ensemble structure-fondation-sol en balancement et en pompage. Elle a un rôle plus important sur les modes de déformation structuraux en diminuant la fréquence de ces modes. L'influence dans un cas correspondant à une centrale nucléaire semble assez peu significative dans l'ensemble.

\section{FONDATION PROFONDE FLEXIBLE}

Le même type d'analyse que précédemment peut être envisagé. Le cas des groupes de pieux est intéressant car il fait intervenir le couplage entre plusieurs fondations flexibles. Afin de simplifier la présentation, les pieux sont supposés encastrés dans un radier rigide. Le déplacement du radier rigide $\Omega$, s'écrit encore comme précédemment :

$$
u_{f}=\sum_{m} c_{m} L_{m} \quad m=1,6
$$

Il est possible d'obtenir une expression équivalente à (45) en appliquant le principe des travaux virtuels avec un mouvement rigidifiant non seulement à $\Omega_{f}$ mais à l'ensemble $\left\{\left\{\cup_{3} \Omega_{3}\right\} \cup \Omega_{4}\right\}$. Cela donne une expression plus symétrique et qui fait disparaître l'interaction entre ces deux parties. Il est commode pour cela de poset $\Omega_{\mathrm{fG}}=\Omega_{\mathrm{f}} \cup\left\{\cup_{\mathrm{J}} \Omega_{\mathrm{J}}\right\}$. Le principe des travaux virtuels appliqué alors à $\Omega_{\mathrm{fG}}$ s'écrit :

$$
\begin{aligned}
& -\omega^{2} \sum_{m} c_{m}\left(\rho_{f} L_{m}, L_{n}\right)_{f G} \\
& =-\left\langle t_{s}\left(u_{s}\right), L_{n}\right\rangle_{s f G}-\left\langle t_{b}\left(u_{b}\right), L_{n}\right\rangle_{b f}
\end{aligned}
$$

Le principe des travaux virtuels appliqué au pieu $J$ s'écrit en choisissant un déplacement virtuel $v_{j}$ de ce pieu respectant la condition d'encastrement en tête :

$$
\begin{aligned}
& -\omega^{2}\left(\rho_{f} u_{j}, v_{j}\right)+\left(\sigma_{j}\left(u_{j}\right), \varepsilon\left(v_{j}\right)_{j}\right. \\
& =-\left\langle t_{s}\left(u_{s}\right), v_{j}\right\rangle_{s j}
\end{aligned}
$$

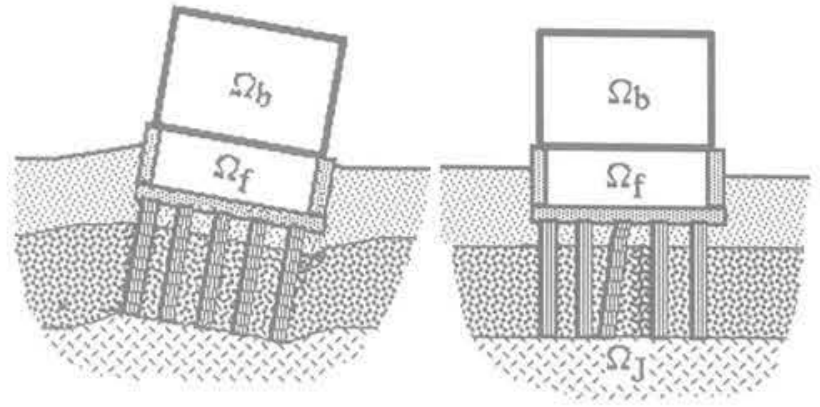

Fig. 4. - Champs diffractés par le groupe de pieux.

L'introduction des modes propres des pieux dans le vide et encastrés sur la fondation est encore fructueuse. Afin de dégager les termes essentiels sans trop compliquer les équations, nous exprimerons les propriétés de ces modes sur la forme tridimensionnelle du principe des travaux virtuels. L'expression détaillée des divers termes s'obtient évidemment en tenant compte de cette hypothèse. Les modes de vibration d'un pieu vérifient une équation semblable à ceux de la structure. Appelons $\phi^{j /}$ et $\omega_{\text {Jj }}$ respectivement le mode j et la fréquence propres associés du pieu J. Ce couple vérifie les équations suivantes :

$$
\begin{aligned}
& \left(\sigma_{J}\left(\phi^{j j}\right), \varepsilon\left(v_{J}\right)\right)_{J}=\omega_{J j}{ }^{2}\left(\rho_{J} \Phi^{j j}, v_{J}\right)_{J} \\
& \phi^{J j}=0 \quad \operatorname{sur} \Gamma_{J f}
\end{aligned}
$$

pour tout déplacement virtuel du pieu $v_{j}$ cinématiquement admissible. En normalisant ces modes propres, on a encore :

$$
\left(\sigma_{j}\left(\Phi^{j i}\right), \varepsilon\left(\Phi^{j i}\right)\right)_{j}=\omega_{j j}{ }^{2} \delta_{j i}
$$

où $\delta$ désigne le symbole de Kronecker. On peut alors écrire en utilisant (33):

$$
u_{j}=\sum_{m} c_{m} L_{m}+\sum_{j j} \alpha_{j j} \phi^{J j}
$$

On peut enfin reporter cela dans l'expression des travaux virtuels en tenant compte de (14):

$$
\begin{aligned}
& -\omega^{2} \sum_{m} c_{m}\left(\rho_{J} L_{m}, \phi^{j i}\right)_{J}+\left(\omega_{J j}^{2}-\omega^{2}\right) \alpha_{J j} \\
= & -\left\langle t_{s}\left(u_{s}\right), \phi^{J i}\right\rangle_{s J}
\end{aligned}
$$

Divers champs rayonnés qui vont généraliser la décomposition doivent encore être introduits. On écrit alors :

$$
\begin{array}{ll}
u_{s}=u_{i}+u_{d o}+\sum_{m} c_{m} u_{d m} & \\
+\sum_{j j} \alpha_{j j} u_{d j j} & \text { dans } \Omega_{s}
\end{array}
$$

Ces champs sont tous des champs de l'élastodynamique stationnaire vérifiant les équations de Navier dans le sol, les conditions de surface libre sur $\Gamma_{5 a}$ et de radiation sur $\Gamma_{s \infty}$. Ils diffèrent seulement sur $\left\{\cup{ }_{j} \Gamma_{s, j} \cup \Gamma_{s i}\right.$ : 
(58)

$$
\begin{array}{ll}
u_{d o}=-u_{i} & \operatorname{sur}\left\{\cup { } _ { J } \Gamma _ { s d } \left\{\cup \Gamma_{s f}\right.\right. \\
u_{d m}=L_{m} & \operatorname{sur}\left\{\cup \Gamma_{s J}\right\} \cup \Gamma_{s t} \\
u_{d J j}=\phi J & \operatorname{sur} \Gamma_{s J} \\
u_{d J\}}=0 & \operatorname{sur}\left\{\cup_{K} \Gamma_{s K}\right\} \cup \Gamma_{s f} \quad K \neq J
\end{array}
$$

Physiquement chacun de ces champs correspond soit au mouvement d'ensemble rigidifiant du radier et des pieux, soit à la flexibilité du pieu $\mathrm{J}$ alors que le radier et les autres pieux sont fixes.

\subsection{Mouvement de la fondation rigide et du groupe}

$$
\begin{aligned}
& \sum_{m} c_{m}\left\{-\omega^{2}\left(\rho_{t} L_{m}, L_{n}\right)_{f G}\right. \\
& +<t_{s}\left(u_{d m}\right), L_{n}>_{s f G\}} \\
& +\sum_{A} \alpha_{b A}<t_{b}\left(\phi^{b A}\right), L_{n}>_{b i} \\
& +\sum_{j,} \alpha_{J j}<t_{s}\left(u_{d j j}\right), L_{n}>_{s i G} \\
& =-<t_{s}\left(u_{1}+u_{d o}\right), L_{n}>_{s i G} \quad n=1,6
\end{aligned}
$$

\subsection{Mouvement du pieu J}

$$
\begin{aligned}
& \text { (60) } \quad \sum_{m} c_{m}\left\{-\omega^{2}\left(\rho_{j} L_{m}, \phi^{J}\right)_{j}\right. \\
& \left.\left.+\left\langle\mathrm{t}_{s}\left(\mathrm{u}_{\mathrm{dm}}\right), \phi^{J}\right\rangle\right\rangle_{\text {sJ }}\right\} \\
& \left.+\left(\omega_{j}{ }^{2}-\omega^{2}\right) \alpha_{j j}+\sum_{\mathrm{li}} \alpha_{\mathrm{li}}<\mathrm{t}_{\mathrm{s}}\left(\mathrm{u}_{\mathrm{dti}}\right), \phi^{\mathrm{j} j}\right\rangle_{\mathrm{s}, \mathrm{j}} \\
& =-\left\langle\mathrm{t}_{\mathrm{s}}\left(\mathrm{u}_{1}+\mathrm{u}_{\mathrm{do}}\right), \Phi^{\mathrm{J}}\right\rangle_{s \mathrm{~J}} \mathrm{j}=1, \mathrm{~N}_{\mathrm{J}} \mathrm{J}=1, \mathrm{~N}_{\mathrm{p}}
\end{aligned}
$$

$\mathrm{Si}$ on pose successivement :

$$
\begin{aligned}
& \mathrm{F}^{*}{ }_{\mathrm{n}}=-\left\langle\mathrm{t}_{\mathrm{s}}\left(\mathrm{u}_{1}+\mathrm{u}_{\mathrm{do}}\right), \mathrm{L}_{\mathrm{n}}\right\rangle_{\mathrm{siG}} \\
& \mathrm{F}^{\mathrm{G} \cdot{ }_{\mathrm{n}}}=-\left\langle\mathrm{t}_{\mathrm{s}}\left(\mathrm{u}_{1}+\mathrm{u}_{\mathrm{do}}\right), \phi_{\mathrm{j}}\right\rangle_{\text {s.j }} \\
& \mathrm{K}^{\mathrm{fG}} \mathrm{jjn}_{\mathrm{j}}=\left\langle\mathrm{t}_{\mathrm{s}}\left(\mathrm{u}_{\mathrm{dj} j}\right), \mathrm{L}_{\mathrm{n}}\right\rangle_{\mathrm{s} f \mathrm{G}} \\
& \mathrm{K}_{\mathrm{mJj}}^{\mathrm{Gi}}=\left\langle\mathrm{t}_{\mathrm{s}}\left(\mathrm{u}_{\mathrm{dm}}\right), \phi^{\mathrm{Jj}}\right\rangle_{\mathrm{s} J} \\
& \mathrm{~K}^{\mathrm{GG}} \mathrm{HiJj}_{\mathrm{i}}=\left\langle\mathrm{t}_{\mathrm{s}}\left(\mathrm{u}_{\mathrm{dil}}\right), \phi^{\mathrm{dj}}\right\rangle_{\mathrm{s}}
\end{aligned}
$$

les équations (59) et (60) sont équivalentes au système suivant, qui généralise (20):

$$
\begin{aligned}
& {\left[-\omega^{2}\left\{M_{f}+M_{b} \cdot+K\right] c+K^{i G} \alpha=F^{*}\right.} \\
& {\left[-\omega^{2} M^{G f}+K^{G f}\right] c+\left[-\omega^{2} 1+\Delta+K^{G G}\right] \alpha} \\
& =F^{G} .
\end{aligned}
$$

En éliminant $\alpha$ dans le système précédent, on trouve l'impédance généralisée de la fondation rigide sur l'ensemble sol-pieu sous la forme d'une matrice $6 \times 6$.

$$
\begin{aligned}
& \left\{-\omega^{2}\left\{M_{f}+M_{b}^{*}\right\}\right. \\
& +K-K^{G G}-\omega^{2} I+\Delta+K^{G G\}-1} \\
& \left.\left\{-\omega^{2} M^{G i}+K^{G i}\right\}\right] c \\
& =F^{*}-K^{f G}\left[-\omega^{2} I+\Delta+K^{G G}\right]^{-1} F^{G *}
\end{aligned}
$$

Remarque : Là encore la formule de Green permet de montrer que les matrices $\mathrm{K}^{\mathrm{iG}}$ et $\mathrm{K}^{\mathrm{GH}}$ sont transposées l'une de l'autre.

Remarque sur l'interaction cinématique : On peut encore définir l'interaction cinématique en considérant le cas d'un radier et d'un bâtiment sans masse. On définit alors $c_{1}$ solutions du système (64) lorsque $M_{f}$ et $M_{6}$ * sont nulles, comme étant le mouvement sismique induit du radier rigide sur pieux.

\subsection{Prise en compte de l'élancement des pieux}

Comme dans le cas des plaques, il est intéressant de supposer que les pieux sont suffisamment élancés pour que des hypothèses cinématiques de poutres puissent être utilisées de manière à passer au niveau des pieux d'un problème tridimensionnel à un problème à une dimension. Si on fait une hypothèse de BernouilliEuler, négligeant les déformations de cisaillement devant ceux de flexions, le déplacement du pieu $J$ s'écrira :

$$
\begin{aligned}
& u_{j}\left(x^{\prime}, x_{3}\right)=\sum_{m} c_{m} L_{m}+u_{d_{1}}\left(x_{3}\right) \\
& -\left\{x^{\prime}, \partial_{3} u_{J_{1}^{\prime}}\left(x_{3}\right)\right\} e_{3}
\end{aligned}
$$

où $x^{\prime}$ désigne les variables horizontales et $x_{3}$ l'abscisse verticale le long du pieu $\mathrm{J}$. En injectant l'hypothèse cinématique précédente dans l'expression du travail virtuel du pieu J, et en se restreignant à des déplacements $v_{j}$ cinérnatiquement admissibles, c'est-à-dire respectant l'encastrement avec $\Omega_{f}$ ainsi que la cinématique de Bernoulli, on obtient:

$$
\begin{aligned}
& -\omega^{2} \sum_{m} c_{m}\left(\rho_{p} L_{m}, v_{j}\right)_{j} \\
& -\omega^{2}\left\{\left(\rho_{\mathrm{p}} u_{J_{1}}, v_{J_{1}}\right)_{j}\right. \\
& \left.+\left(\rho_{\mathrm{p}} \mathrm{x}^{\prime} \cdot \partial_{3} u_{\mathrm{d}_{1}}{ }^{\prime}, \mathrm{x}^{\prime} \cdot \partial_{3} v_{\mathrm{J}_{1}}{ }^{\prime}\right)_{\mathrm{J}}\right\} \\
& +\left(\sigma_{\jmath}\left(u_{\jmath}\right), \varepsilon\left(v_{\jmath}\right)\right) \text { 小 } \\
& =-\left\langle t_{s}\left(u_{s}\right), v_{J_{1}}\right\rangle_{s J} \\
& \left.+<t_{5}\left(u_{5}\right), x_{5}^{\prime} \cdot \partial_{3} v_{J_{1}}\right\rangle_{5 s}
\end{aligned}
$$

Grâce à l'hypothèse cinématique, il est toujours possible d'intégrer par rapport à la section du pieu. L'équation précédente devient alors, en supposant les symétries convenables du pieu par rapport à la fibre moyenne :

$$
\begin{aligned}
& -\omega^{2} \sum_{m} c_{m}\left(\rho_{p} L_{m}, v_{j}\right)_{j} \\
& \left.-\omega^{2}\right\} \ll \mathrm{mu}_{J_{1}}, v_{\mathrm{d}_{1}} \gg_{\mathrm{J}} \\
& +\ll\left[\partial_{3} u_{j 1}{ }^{\prime}, \partial_{3} v_{j 1} \gg_{j}\right\} \\
& +\ll n_{\jmath}\left(u_{J_{1}}\right), \varepsilon\left(v_{J_{1}}\right) \gg_{\jmath} \\
& +\ll \mathrm{m}_{\mathrm{J}}\left(\mathrm{u}_{\mathrm{j}_{1}}\right), \mathrm{k}\left(\mathrm{v}_{\mathrm{J}_{1}}\right) \gg_{\mathrm{J}} \\
& =-\left\langle t_{s}\left(u_{5}\right), v_{j_{1}}\right\rangle_{s, j} \\
& +\left\langle\mathrm{t}_{\mathrm{s} 3}\left(\mathrm{u}_{\mathrm{s}}\right), \mathrm{x}_{\mathrm{s}}^{\prime} \cdot \partial_{3} \mathrm{v}_{\mathrm{J}_{1}}\right\rangle_{\mathrm{s}, \mathrm{J}}
\end{aligned}
$$




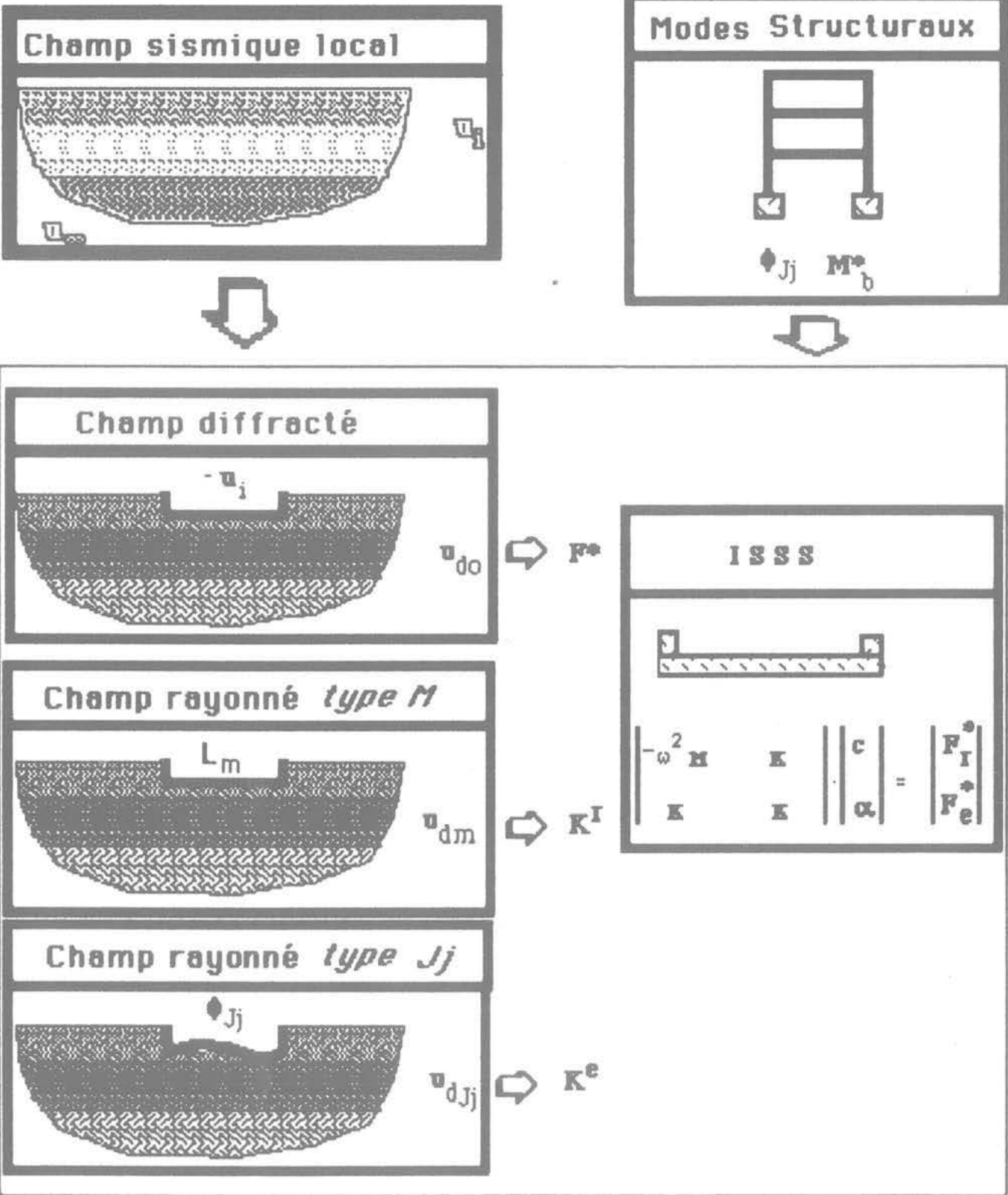

Planche 1. - Modules généraux de calculs d'interaction sol-structure.

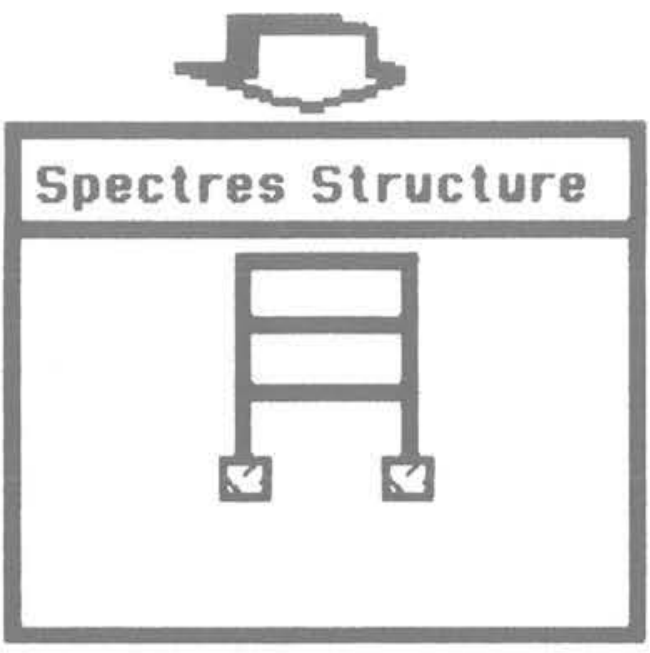


où $\mathrm{l}, \mathrm{m}, \mathrm{n}_{\mathrm{J}}, \mathcal{E}, \mathrm{m}, \mathrm{k}$ désignent respectivement le moment d'inertie du pieu, la masse linéique, les moments de flexion et les courbures du pieu J. Enfin la notation $\ll, \gg$ désigne une intégration curviligne le long du pieu $\mathrm{J}$.

Les termes du membre de gauche de (65) sont classiques du point de vue mécanique des structures. Les modes propres des pieux utilisés dans le système de l'ISSS respecteront la cinématique précédente, qui conduira par conséquent à développer les intégrations par rapport à la section des pieux. Il est intéressant d'expliciter le terme de droite. On trouve alors :

$$
\begin{aligned}
& <t_{s}\left(u_{s}\right), v_{J_{1}}>_{s j} \\
& =\ll T_{s}\left(u_{s}\right), v_{J_{1}} \gg \\
& \left\langle t_{s 3}\left(u_{s}\right), x^{\prime} \cdot \partial_{3} v_{d_{1}}^{\prime}\right\rangle_{s, d} \\
& =\ll M_{s}^{\prime}\left(u_{s}\right), \partial_{3} v_{J_{1}}^{\prime} \gg
\end{aligned}
$$

où $T_{\text {s }}$ et $M_{s}^{\prime}$ désignent respectivement l'effort tranchant et le moment de flexion exercés par le sol sur le pieu J. Ces termes de droite matérialisent l'interaction solstructure et mettent en évidence les sollicitations venant du sol adaptées à la cinématique du pieu. Il est important de remarquer que la sollicitation sismique appliquée au pieu est donnée par :

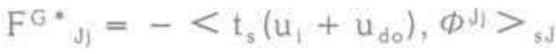

$$
\begin{aligned}
& =\ll \mathrm{T}_{\mathrm{s}}\left(\mathrm{u}_{1}+\mathrm{u}_{\mathrm{do}}\right), \phi^{J_{j}} \gg \\
& +\ll M_{s}^{\prime}\left(u_{i}+u_{d o}\right), \partial_{3} \phi^{j^{\prime}} \gg
\end{aligned}
$$

C'est donc la somme du champ libre et du champ diffracté qui doit être appliquée au pieu. Si on applique seulement l'action développée par le champ libre, on néglige l'interaction sol-structure.

\subsection{Quelques remarques}

sur le comportement dynamique

des groupes de pieux

$[5][8][17][18][19][22][58][59][60][61]$

[63][66] [68] [70] [73] [78] [79]

La réponse dynamique d'un groupe de pieux dépend beaucoup de la fréquence à cause des interférences qui se développent. En ce qui concerne le pompage et le tamis, si l'espacement entre les pieux est très faible, l'impédance normalisée par l'impédance statique varie en fonction de la fréquence comme celle d'une fondation rigide en deçà d'une certaine fréquence. Les rigidités décroissent avec la fréquence et deviennent même négatives, indiquant un comportement où l'accession à l'inertie est importante.

L'amortissement dans ce cas varie peu. Lorsque la fréquence dépasse cette limite, l'interaction entre les pieux devient dominante. La valeur de cette fréquence diminue lorsque l'espacement augmente. L'amortissement augmente en général avec l'espacement. Lorsque le nombre de pieux augmente, l'effet de groupe est encore plus prononcé et la variation de l'impédance est plus importante. L'interaction est alors très forte, plus marquée pour le pompage que pour le tamis et met en jeu des vibrations des pieux non en phase. Lorsque la rigidité du sol augmente, l'interaction est moins importante.

En ce qui concerne le balancement et la torsion, des résultats semblables sont obtenus avec une interaction plus forte. Du point de vue de l'hétérogénéité du sol, dans le cas d'une couche très molle superficielle lorsque le nombre de pieux augmente, l'impédance est très proche de celle du demi-espace : par conséquent, des groupes de pieux sont moins influencés par des conditions locales qu'un seul pieu. Du point de vue de la réponse sismique, des ondes $\mathrm{SH}$ verticales produisent à la fois tamis et balancement du groupe; la fonction de transfert du mouvement horizontal est proche de l'unité à basse fréquence, donc le mouvement sismique induit horizontal est très proche de celui du sol. Par contre le balancement diminue rapidement avec l'espacement. Pour un sol plus rigide la fonction de transfert est plus longtemps proche de l'unité. Les fréquences plus élevées sont en général filtrées par le groupe de pieux. Dans le cas d'ondes inclinées, la torsion augmente.

La répartition des charges dans les pieux est un paramètre de dimensionnement très important : à basse fréquence, ce sont les pieux placés aux extrémités qui sont les plus chargés; lorsque la fréquence augmente ce phénomène peut être inversé; les effets dynamiques sont plus marqués en pompage qu'en tamis. Les solutions simplifiées et en particulier les hypothèses "quasi tridimensionnelles" de Novak sont des solutions pour lesquelles on se ramène à une cinématique unidirectionnelle du sol; cela donne de bons résultats en général; la méthode de superposition de Poulos où seulement deux pieux sont considérés à la fois donne également des résultats convenables.

\section{LES MODULES DE CALCULS}

Les développements précédents ont permis d'aboutir dans chaque cas au système de l'ISSS que ce soit dans le cas d'une fondation rigide, d'un radier flexible ou de fondation profonde élancée telle que des pieux. Dans chaque cas en utilisant des champs globaux diffractés et rayonnés au niveau du sol, ainsi que les modes de la structure et de la partie flexible de la fondation, nous avons pu construire ce système. La méthodologie a consisté dans tous les cas à ramener tout au niveau de la fondation qui constitue une interface privilégiée entre la structure et le sol. Cette approche permet de ne pas préjuger des techniques numériques permettant de calculer les divers champs de déplacements invoqués plus haut. En particulier cela permet d'utiliser la méthode la plus adaptée à chaque système et donc du point de vue de la mise en ceuvre informatique de construire des codes modulaires avec des données et des sorties bien définies. Essentiellement les ressources suivantes doivent être développées :

- un code de calcul du champ sismique local $u_{1}$ en champ libre;

- un code de calcul des champs $u_{d o}, u_{d m}, u_{j\}}$ résolvant les équations de Navier dans un sol hétérogène non borné. Les données de ce code seront $u_{1}, L_{m}, \Phi_{j}$ le long de l'interface sol-fondation. Les résultats fournis 
par ce code seront les impédances généralisées $K$ et les forces sismiques $\mathrm{F}^{*}$ qui consistent à calculer diverses intégrales sur $\Gamma_{5 f}$;

- un code de calcul des structures permettant de calculer les modes propres de la structure sur base fixe $\Phi^{b}$ et la matrice de masse équivalente $M_{b}{ }^{*}$ de la structure :

- un code d'ISSS mettant en place et résolvant le système ISSS. Ce code fournit les déplacements c de la partie rigide de la fondation, à laquelle la structure est connectée. Le code de calcul des structures à partir des déplacements de la base peut aisément ensuite calculer les déplacements de celle-ci et les efforts correspondants.

Les efforts calculés dans la structure sont donnés dans le domaine des fréquences. Une transformation de Fourier permet par passage dans le temps de calculer les efforts maximums en un certain nombre de points de contrôle. La figure ci-dessous résume les étapes du calcul et les interfaces entre les différents modules.

Dans l'analyse qui vient d'être présentée, les termes qui sont relatifs à la structure peuvent être obtenus de manière standard par une méthode d'éléments finis. Le calcul des champs diffractés dans le sol est cepen. dant moins standard et également très coûteux si l'on veut garder le caractère souvent tridimensionnel du problème. Nous examinons dans la section suivante quelles sont les méthodes qui semblent les plus efficaces pour traiter ces problèmes.

\section{CALCUL DES CHAMPS DIFFRACTÉS DANS LE SOL}

De nombreuses méthodes ont été proposées dans la littérature allant des développements presque entièrement analytiques jusqu'à des méthodes d'éléments finis avec des possibilités de méthodes hybrides. Si l'on peut faire une hypothèse de sol hétérogène avec couches horizontales, il semble qu'à l'heure actuelle, une méthode d'équations intégrales utilisant la fonction de Green du sol multicouche soit la plus compétitive. Lorsque cette hypothèse ne peut être tenue au voisinage de la fondation, alors qu'elle convient encore à quelques distances, il faut envisager un couplage entre cette technique et une méthode d'éléments finis. Nous discutons ci-dessous ces deux cas succinctement.

\subsection{Sol à stratigraphie horizontale}

Le problème de l'ISSS a été ramené aux calculs successifs de champs de déplacements dans le sol vérifiant les équations de Navier de l'élastodynamique, des conditions de surface libre, des conditions de continuité à l'interface entre les couches et des conditions de radiation à l'infini. Ces dernières conditions sont certainement les plus difficiles à respecter en particulier si on veut utiliser une technique d'éléments finis. Une méthode d'équations intégrales permet de prendre en compte automatiquement ces conditions. La mise en œuvre procède en deux étapes

- calcul de la fonction de Green,

- mise en ceuvre de la méthode intégrale.

\section{Calcul de la fonction de Green.}

La fonction de Green est un champ de déplacements $\mathrm{u}^{\mathrm{Gk}}(\mathrm{x}, \xi)$ dans le demi-espace stratifié, naturel, solution des équations de Navier avec les conditions aux limites, internes, et de radiation, mentionnées précédemment. Cette fonction $u^{G k}(x, \xi)$ donne le déplacement pour un observateur placé au point $x$ pour une force concentrée placée au point $\xi$ dans la direction $e_{k}$.

Dans le cas où le sol est viscoélastique isotrope, il est possible de calculer $u^{\text {Gk }}$ d'une manière très efficace, en utilisant une transformée de Hankel. Toutes les équations de l'élastodynamique sont résolues dans le domaine transformé - le domaine spectral. La transformation de Hankel ramène les équations de Navier à des équations diffêrentielles par rapport à la variable "verticale" $x_{3}$. La force ponctuelle introduit des discontinuités du vecteur contrainte spectral. Sur le plan théorique la méthode ne pose pas de difficultés. Cependant sur le plan numérique, il convient de traiter avec soin :

- le système différentiel. Seule une technique utilisant des opérateurs de réflexion-transmission permet d'éviter des instabilités bien connues dans le cas de couches épaisses [53]. L'algorithme correspondant suggéré par KENNETT [39] a été développé par TSAKALIDIS dans sa thèse [21] [23] [73];

- la transformée inverse effectuée numériquement si l'on veut éviter des couts prohibitifs. Une technique permettant de se ramener à une transformée de Fourier rapide et une intégration numérique est également présentée dans la thèse de TSAKALIDIS. L'avantage de cette méthode est qu'elle peut être vectorisée sur des machines vectorielles comme le Cray-1. L'arrivée de machines parallèles comme le Cray- 2 permettra des temps de calculs encore plus compétitifs.

\section{Équation intégrale de surface.}

La fonction de Green du sol naturel multicouche étant connue numériquement, elle peut être utilisée pour établir une équation intégrale de surface (CHAPEL. [17], KUPRADZE [43], WOLF et al. [81] [82]). Grâce à la formule de Green en prenant soin de bien traiter la singularité, on obtient classiquement :

$$
\begin{aligned}
k u_{k}^{\prime}(\xi)= & \left\langle t\left(u^{\prime}\right), u^{G k}\right\rangle_{r} \\
& -\left\langle u^{\prime}, t\left(u^{G k}\right)\right\rangle_{r}
\end{aligned}
$$

où $\mathrm{k}$ est une constante dépendant des caractéristiques de la frontière et pour alléger les notations nous avons posé $\Gamma=\Gamma_{\mathrm{fs}}$. $u^{\prime}$ est un des champs diffractés $u_{d o}, u_{d m} \ldots$ L'avantage d'avoir calculé au préalable la fonction de Green est que les conditions aux limites de surface libre et internes entre les couches sont prises en compte dans la formulation.

Dans tous les sous-problèmes envisagés ci-dessus le champ u' est donné sur $\Gamma_{t \mathrm{~s}}$. Donc $(66)$ est une équation intégrale où $t\left(u^{\prime}\right)$ est la seule inconnue. On peut réécrire (66) sous la forme suivante :

$$
\begin{array}{r}
\left\langle t\left(u^{\prime}\right), u^{G k}\right\rangle_{r}=k u_{k}^{\prime}(\xi)+\left\langle u^{\prime}, t\left(u^{G k}\right)>_{r}\right. \\
\xi \in \Gamma
\end{array}
$$


La frontière $\Gamma$ est alors triangulée en $\mathrm{N}_{e}$ éléments triangulaires ou quadrilatéraux E. Dans le cas le plus simple, on approche le champ $t\left(u^{\prime}\right)$ par des fonctions constantes par élément $w_{E_{1}}$, où $E$ fait référence au numéro d'élément et i à la direction d'espace. Si bien qu'on peut écrire :

$$
t\left(u^{\prime}\right)=\sum_{E, 1} t_{E,} w_{E l}(x)
$$

Cette expression approchée est ensuite injectée dans (67) :

$$
\begin{aligned}
& \left.\sum_{E, j} t_{E i}<w_{E i}, u^{G k}\right\rangle_{T} \\
& =k u_{k}^{\prime}(\xi)+\left\langle u^{\prime}, t\left(u^{G k}\right)\right\rangle_{r} \quad \xi \in \Gamma
\end{aligned}
$$

Les fonctions $w_{E_{1}}$ sont normalisées à 1 sur l'élément $E$ dans la direction $\mathrm{i}$, et sont nulles sur les autres éléments et dans les autres directions. D'autre part (68) est une équation fonctionnelle en $\xi$. Pour se ramener à un système algébrique, on impose à (68) d'être vérifiée successivement au centre de gravité $\xi_{F}$ de chaque élément $\mathrm{F}$. Ce qui donne finalement en tenant compte des propriétés des fonctions de base $\mathrm{w}_{\mathrm{E}}$ un système linéaire de $3 \mathrm{~N}_{e}$ équations à $3 \mathrm{~N}_{\mathrm{e}}$ inconnues :

$$
\begin{aligned}
& \left.\quad \sum_{E, i} t_{E i}<u^{G k}\left(x, \xi_{F}\right)\right\rangle_{E} \\
& =k u_{k}^{\prime}\left(\xi_{F}\right)+<u^{\prime}, t\left(u^{G k}\left(x, \xi_{F}\right)\right)>_{r} \\
& \quad k=1,3 ; F=1, N_{e}
\end{aligned}
$$

Lorsque $F=E$, le coefficient de $t_{F}$ est singulier ; il doit être calculé au sens de la valeur principale de Cauchy. La matrice du système est la même pour tous les sousproblèmes envisagés, seul le second membre est modifié. Une fois résolu le système linéaire précédent, il faut ensuite calculer les diverses intégrales qui interviennent dans le calcul des impédances. Par exemple:

$$
\begin{aligned}
K_{m n} & =\left\langle t_{s}\left(u_{d m}\right), L_{n}\right\rangle_{r} \\
& \left.=\sum_{E, 1} t^{m}<L_{n i}\right\rangle_{E}
\end{aligned}
$$

où $\mathrm{t}^{\mathrm{m}} \mathrm{E}$ désigne les vecteurs contraintes approchés obtenus dans la résolution lorsqu'au second membre, ' ' $^{\prime}$ est égale à $\mathrm{L}_{n}$ le long de la fondation. On peut remarquer ici que dans le calcul de l'impédance, ce n'est pas la distribution précise du vecteur contrainte qui compte, mais sa moyenne. Cela est important car dans le cas du sol hétérogène, la fonction de Green n'est pas connue analytiquement mais seulement numériquement. Un calcul précis des champs exige de nombreuses évaluations de cette fonction. De nombreuses variations à l'approche qui est présentée ici existent (WOLF [81]).

\subsection{Sol à stratigraphie quelconque (flexible volume element)}

Lorsque la stratigraphie n'est plus homogène horizontalement, il est souvent encore possible de se limiter à des hétérogénéités au voisinage de la fondation. Un couplage entre une méthode intégrale et une méthode d'éléments finis est intéressante. On partitionne alors
$\Omega_{5}$ en deux parties : le domaine intérieur $\Omega$ irrégulier et le domaine extérieur $\Omega^{\prime}$ homogène latéralement. Soit $\Sigma$ la frontière entre ces deux domaines.

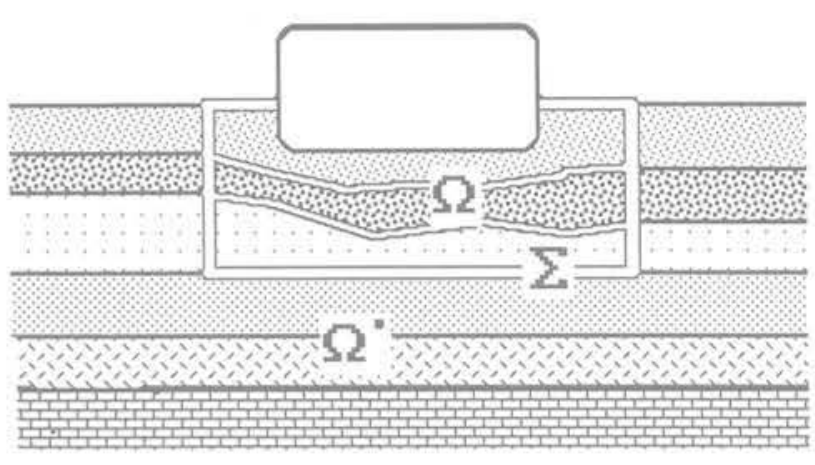

Fig. 5. - Couplage éléments finis - équations intégrales.

La formulation variationnelle dans le domaine intérieur et les conditions de continuité le long de $\Sigma$ s'écrivent, en appelant u le déplacement dans le domaine intérieur et $\mathrm{u}^{\prime}$ dans le domaine extérieur :

$$
\begin{aligned}
& -\omega^{2}(\rho u, v)_{\Omega} \\
& +(\sigma(u), \varepsilon(v))_{\Omega}-<t(u), v>\Sigma=0 \\
& t(u)+t^{\prime}\left(u^{\prime}\right)=0 \quad \operatorname{sur} \Sigma \\
& u=u^{\prime} \quad \operatorname{sur} \Sigma
\end{aligned}
$$

En tenant compte de $(69)_{2}$ dans $(69)_{1}$ et en imposant la continuité des déplacements seulement au sens des travaux virtuels, pour tout $v$ et $v^{\prime}$ cinématiquement admissibles, on peut écrire :

$$
\begin{aligned}
& -\omega^{2}(\rho u, v)_{\Omega} \\
& +(\sigma(u), \varepsilon(v))_{\Omega}+\left\langle t^{\prime}\left(u^{\prime}\right), v\right\rangle \Sigma=0 \\
& \left\langle u, t^{\prime}\left(v^{\prime}\right)\right\rangle \Sigma-\left\langle u^{\prime}, t^{\prime}\left(v^{\prime}\right)\right\rangle \Sigma=0
\end{aligned}
$$

Dans le domaine intérieur, on cherche une solution approchée $u_{h}$, à l'aide d'une méthode d'éléments finis, en appelant $w_{11}$ la fonction de base attachée au nœud I, dans la direction $\mathrm{i}$ :

$$
u_{h}=\sum_{11} u_{11} w_{11}
$$

En injectant cette expression dans $(69)_{1}$, on construit l'impédance éléments finis du domaine intérieur.

Remarque : En utilisant des fonctions de base $\Phi_{\mathrm{m}}$ vérifiant exactement les équations de l'élastodynamique, on peut obtenir une expression intéressante de l'impédance discrète et symétrique d'un hyperélément $\mathrm{E}$ de frontière $\partial E$ :

$$
\begin{aligned}
& -\omega^{2}\left(\rho \Phi_{m}, \Phi_{n}\right)_{E}+\left(\sigma\left(\Phi_{m}\right), \mathcal{E}\left(\Phi_{n}\right)\right)_{E} \\
& =-\omega^{2}\left(\rho \Phi_{m}, \Phi_{n}\right)_{E} \\
& -\left(\operatorname{Div} \sigma\left(\Phi_{m}\right), \Phi_{n}\right)_{E}+\left\langle t\left(\Phi_{m}\right), \Phi_{n}\right\rangle_{\partial E} \\
& =\left\langle t\left(\Phi_{m}\right), \Phi_{n}\right\rangle_{\partial E}
\end{aligned}
$$


En utilisant cette idée, on peut aisément envisager le couplage entre une méthode d'équations intégrales pour le domaine extérieur et une méthode d'éléments finis pour le domaine intérieur. Pour cela, dans un premier temps, à chaque fonction de base $w_{11}$ d'un nœud de l'interface, on associe le champ rayonné $w_{1 ;}^{\prime}$ tel que $w_{11}^{\prime}$ vérifie les équations de Navier dans le domaine extérieur ainsi que les conditions de radiation et qui enfin vérifie l'équation de raccord suivante sur $\Sigma$ :

$$
\mathrm{w}_{\mathrm{II}}^{\prime}=\mathrm{w}_{\mathrm{H}} \quad \text { sur } \Sigma
$$

Si enfin, on utilise l'équation (71), pour définir l'impédance "éléments finis» symétrique du domaine $\Omega$ ', on obtient la rigidité dynamique du domaine extérieur :

$$
\mathrm{K}_{(\mathrm{wj})}=\left\langle\mathrm{t}^{\prime}\left(\mathrm{w}_{\mathrm{ji}}^{\prime}\right), \mathrm{w}_{\mathrm{j}}^{\prime}\right\rangle \Sigma
$$

$w_{\text {H }}^{\prime}$ est déterminé par une méthode d'équation intégrale mise en œuvre comme ci-dessus, $u_{h}^{\prime}$ est développé également sur cette base. (59) 2 est identiquement vérifié. En reportant (73) dans (70), on obtient dans un deuxième temps, le système linéaire éléments finis à résoudre :

$$
\begin{aligned}
& -\omega^{2} \sum_{11} u_{11}\left\{\left(\rho w_{11}, w_{j j}\right)_{\Omega}\right. \\
& +\left(\sigma\left(w_{1 i}\right), \varepsilon\left(w_{11}\right)\right)_{\Omega}+\left\langle t^{\prime}\left(w_{11}^{\prime}\right), w_{j\}}^{\prime}>\Sigma\right\}=0
\end{aligned}
$$

La matrice symétrique $\mathrm{K}$ qui constitue la matrice de rigidité dynamique du super-élément extérieur peut être aisément incorporée dans tout code éléments finis standard. Le calcul du champ sismique libre u, est également complexe mais peut être déterminé par la même procédure.

\section{DÉCOLLEMENT ET GLISSEMENT DE LA FONDATION}

Lorsque les non-linéarités sont limitées à l'interface avec la fondation, il n'est plus possible de rester dans le domaine des fréquences mais une méthode d'équations intégrales est encore très intéressante car les équations sont limitées à cette interface. Il faut alors développer la fonction de Green transitoire du sol naturel stratigraphié $\mathrm{u}^{\mathrm{Gk}}(\mathrm{x}, \mathrm{t} ; \xi, \tau)$ qui vérifie les équations suivantes de l'élastodynamique, où $\Delta^{*}$ est l'opérateur de l'élastostatique :

$$
\Delta^{*} u^{G k}+\delta_{(\xi, 7)} e_{k}=\rho \partial^{2} u^{G k}
$$

Cela peut se faire en faisant une transformée de Fourier inverse de la fonction de Green obtenue en fréquence. Pour une distribution de force quelconque sur l'interface $\Gamma_{f_{5}}$, le déplacement u s'écrit :

$$
\mathrm{u}_{\mathrm{k}}(\xi, \tau)=\int_{\mathrm{R}} \mathrm{dt}\left\langle\mathrm{g}(\mathrm{x}, \mathrm{t}), \mathrm{u}^{\mathrm{Gk}}(\mathrm{x}, \mathrm{t} ; \xi, \tau)\right\rangle_{\text {is }}
$$

En général, on détermine $u^{G^{\circ} k}(x, t ; \xi)=u^{G k}(x, t ; \xi, 0)$. La formule précédente s'écrit alors :

$$
\mathrm{u}_{\mathrm{k}}(\xi, \tau)=\int_{0}^{\mathrm{t}} \mathrm{dt}\left\langle\mathrm{g}(\mathrm{x}, \mathrm{t}), \mathrm{u}^{\mathrm{G}^{\circ} \mathrm{k}}(\mathrm{x}, \mathrm{t}-\tau ; \xi)\right\rangle_{\text {is }}
$$

Si $\xi \in \Gamma_{\text {is }}$, comme dans le cas stationnaire, on obtient une équation intégrale :

$$
\begin{aligned}
& \mathrm{ku}_{\mathrm{k}}(\xi, \tau)= \\
& \int_{0}^{\tau} \mathrm{dt}\left\{\left\langle\mathrm{t}(\mathrm{u}), \mathrm{u}^{\mathrm{G}^{\circ} \mathrm{k}}\right\rangle_{\text {is }}\right. \\
& \left.\left.-<\mathrm{u}, \mathrm{t}\left(\mathrm{u}^{\mathrm{G} \mathrm{o}^{\mathrm{k}}}\right)\right\rangle_{\mathrm{is}}\right\} \quad \xi \in \Gamma_{i s}
\end{aligned}
$$

La loi de frottement de Coulomb à l'interface permet d'envisager la plupart des cas pratiques. Soit $n$ la normale extérieur au domaine de sol, on introduit :

$$
t_{N}=t \cdot n, t_{T}=t-t_{N} n
$$

et des notations identiques pour le déplacement $u$. Alors la loi de Coulomb s'écrit avec F le coefficient de frottement à l'interface sol-fondation :

$$
u_{f N}-u_{s N} \geq 0 \text {, }
$$

Si $u_{i N}-u_{s N}>0$, alors : $t\left(u_{s}\right)=0$

Si $u_{f N}-u_{s N}=0$, alors : $t_{N}\left(u_{s}\right) \leq 0$

et si : $\left\|t_{T}\left(u_{s}\right)\right\|<F\left|t_{N}\left(u_{s}\right)\right|$,

alors : $\partial_{1} u_{1 T}-\partial_{1} u_{5 T}=0$

ou si : $\left\|\mathrm{t}_{\tau}\left(\mathrm{u}_{\mathrm{s}}\right)\right\|=\mathrm{F}\left|\mathrm{t}_{\mathrm{N}}\left(\mathrm{u}_{\mathrm{s}}\right)\right|$,

alors : $\exists \lambda \geq 0, \partial_{1} u_{i T}-\partial_{1} u_{s T}=\lambda t_{T}$

Si on tient compte du mouvement de corps rigide de la fondation, les inégalités précédentes peuvent être remplacées par le développement de celui-ci sur la base des déplacements $L_{m}$. L'équation du mouvement transitoire de la fondation rigide et du déplacement du sol s'écrivent :

$$
\begin{aligned}
& \sum_{m} d_{n}{ }^{2} c_{m}\left(\rho_{t} L_{m}, L_{n}\right)_{f}= \\
& -<t_{s}\left(u_{s}\right), L_{n}>_{s t}-\left\langle t_{b}\left(u_{b}\right), L_{n}>_{b f}\right. \\
& \qquad n=1,6 \\
& k u_{k}(\xi, \tau)=\int_{0}^{r} d t\left\{<t(u), u^{G^{\circ} k}>_{r}\right. \\
& \left.-<u, t\left(u^{\circ} k\right)>_{r}\right\} \quad \xi \in \Gamma
\end{aligned}
$$

On ne peut plus développer $\mathrm{u}_{\mathrm{s}}$ comme un déplacement rigide, car au moment du décollement, seule-

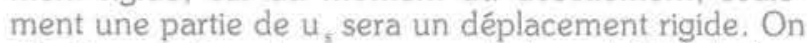
développe alors le mouvement du sol à l'interface selon les fonctions de base utilisées plus haut :

$$
u_{s}=\sum_{E 1} u_{E 1}(t) w_{E 1}, t\left(u_{s}\right)=\sum_{E i} t_{E I}(t) w_{E t}
$$

Ces expressions injectées dans l'équation intégrale de surface donnent, en prenant des fonctions de base constantes par élément :

$$
\begin{aligned}
& \mathrm{ku}_{\mathrm{Fk}}(\tau)= \\
& \int_{0}^{\tau} \mathrm{dt}\left\{\sum_{E_{i}} \mathrm{t}_{E !}(\mathrm{t})<\mathrm{u}^{\mathrm{G}^{\circ} \mathrm{k}}\left(\mathrm{x}, \mathrm{t}-\tau ; \xi_{\mathrm{F}}\right)>_{E}\right. \\
& -\sum_{E_{i}} \mathrm{u}_{E_{i}}(\mathrm{t})<\mathrm{t}_{1}\left(\mathrm{u}^{\mathrm{G}^{\circ} \mathrm{k}}\left(\mathrm{x}, \mathrm{t}-\tau ; \xi_{\mathrm{F}}\right)\right) \\
& \left.\mathrm{F}=1, \mathrm{~N}_{E}\right\}
\end{aligned}
$$


Cette équation ainsi que celle du mouvement de la fondation doivent être reportées dans les inéquations traduisant la loi de Coulomb. Elles sont intégrées pas à pas, éventuellement de manière explicite. Les intégrales portant sur l'interface ne doivent porter que sur la partie au contact.

Remarques sur l'influence du décollement [2] [6] [16] [76] [77] [84] [85]

En examinant un certain nombre de modèles de plus en plus complexes. BISCH [16] parvient à un certain nombre de conclusions : la théorie linéaire surestime en général les efforts et la longueur de décollement; le décollement abaisse la rigidité moyenne : la gravitation qui intervient dès qu'une partie décolle, augmente la pseudo-période et augmente la stabilité. Les rotations sont plus fortes mais si le soulèvement reste faible, l'analyse linéaire donne des résultats peu différents du non-linéaire. Le séisme vertical joue un rôle accru. PSYCHARIS montre que seulement le premier mode du bâtiment est réellement affecté par le décollement. L'effet le plus important est surtout sur la période fondamentale qui est considérablement abaissée. Les modes d'ordre plus élevés semblent peu affectés par ce phénomène.

\section{CONCLUSION}

La seule hypothèse de linéarité de l'ensemble du système sol-fondation-structure a permis dans chaque cas de mettre en évidence la structure du système de Pinteraction sol-structure quel que soit le type de fondation superficielle ou profonde, rigide ou flexible, isolée ou multiple. Cette approche permet d'aborder de manière unifiée dans un code de calculs tous les différents aspects. Le rôle du champ sismique incident a été mis en évidence dans le mouvement sismique induit ou dans le second membre des systèmes linéaires. Le champ libre, ainsi que les contraintes qu'il produit doivent être connus le long de l'interface sol-fondation. Le rôle de la structure a été identifié grâce à la notion de masse équivalente qui constitue l'interface avec le calcul des structures. L'approche proposée permet de bien découpler les problèmes et d'utiliser les techniques les plus adaptées pour chaque sous-problème.

En particulier il n'a été fait référence à une technique numérique qu'après avoir établi les équations du système mécanique continu. Lorsqu'une hypothèse de couches horizontales est acceptable, la technique des équations intégrales de surface est particulièrement intéressante surtout si le substratum est très profond et si le problème est complètement tridimentionnel. Le calcul de la fonction de Green du sol stratifié doit alors être très performant. L'arrivée de nouveaux calculateurs parallèles doit diminuer considérablement les temps de calculs. Par ailleurs de nombreuses approximations sont possibles permettant de construire des méthodes simplifiées. Elles n'ont pas été abordées dans ce texte, mais la présentation unifiée doit permettre d'appréhender les conséquences de telles approximations dans toute la chaîne des calculs.

\section{BIBLIOGRAPHIE}

[1] AKI K., RICHARDS P.G. (1980), Quantitative seismology Theory and Methods, Freeman, San Francisco.

[2] AKIYOSHI T. (1982), Soil-pile interaction in vertical vibration induced through a frictional interface, Earthquake Engineering and Structural Dynamics, vol. X, pp. 135-148.

[3] APSEL R.J., LUCO J.E. (1976), Torsional res. ponse of a rigid embedded foundation, J. Engineering Mechanics Division ASCE, vol. CII, pp. 957-970.

[4] AUBRY D., CHAPEL F., CREPEL J.M. (1985), Interaction sol-structure linéaire sur un sol hétéro. gène, Génie Parasismique (V. Davidovici éd.), Presses ENPC, vol. VI, pp. 485-506.

[5] AUBRY D., CHAPEL F., CREPEL J.M. (1985), Calcul sismique de fondations sur pieux, Génie Parasismique (V. Davidovici éd.), Presses ENPC, vol. VI, pp. 533-547.

[6] AUBRY D. CHOUVET D. (1985), Calcul sismique de murs de soutènement, Génie Parasismique (V. Davidovici éd.), Presses ENPC, vol. VIII, pp. 759-771.

[7] AUBRY D., CHOUVET D., MODARESSI H., MOUROUX P. (1985), Local amplification of $a$ seismic incident field through an elastoplastic sedimentary valley, Numerical Methods in Geomechanics (Ed. Kawamoto, Ichikawa), Balkema, pp. 421-428.

[8] AUBRY D., POSTEL M. (1985), Dynamic response of a large number of piles by homogeneiza. tion, 2nd International Conference Earthquake Engineering.

[9] AUBRY D., CHOUVET D. (1985), Analuse sismique de murs de soutènement, International Conference Soil Mechanics, 11th International Conference Soil Mechanics Foundation Engineering, San Francisco, Balkema.

[10] AUBRY D., CRÉPEL J.M. (1986), Interaction sismique fluide-structure. Application aux barrages voute, Actes let Colloque Français de Génie Parasismique, Association Française Génie Parasismique.

[11] BARD P. Y, , BOUCHON M. (1980), The seismic response of sediment-filled valley, Part 1 , The case of incident SH waves, Bull. Seism. Soc. Am., vol. LXX-4, pp. 1236-1286.

[12] BARD P Y . BOUCHON M. (1980), The seismic response of sediment-filled valley, Part 2, The case of incident $P$ and SV waves, Bull. Seism. Soc. Am., vol. LXX-5, pp. 1921-1941.

[13] BARD P.Y. (1982), Diffracted waves and displacement field over two-dimensional elevated topo. graphies, Geophys, J. R. astr. Soc., vol. LXXI, pp. 731-760.

[14] BARD P.Y. (1985), Les effets de site d'origine structurale : principaux résultats expérimentaux et théoriques, Génie Parasismique (V. Davidovici éd.), Presses ENPC, vol. III, pp. 223-238. 
[15] BEREDUGO Y.O., NOVAK M. (1972), Coupled horizontal and rocking vibration of embed. ded footings, Canadian Geotechnical J., vol. IX-4.

[16] BISCH P. (1985), Effets non linéaires des décollements de radier sur le comportement des dynamiques des structures, Génie Parasismique (V. Davidovici éd.), Presses ENPC, vol. VI, pp. 549-560.

[17] CHAPEL F. (1981), Application de la méthode des équations intégrales à la dynamique des sols, Thèse de Docteur Ingénieur, École Centrale de Paris.

[18] CHAPEL F., AUBRY D. (1981), Three dimensional dynamic analysis of a group of piles and comparison with experiments, 6th SMIRT Conference, Paris, K5/7.

[19] CHAPEL F., AUBRY D. (1982), Three dimensional analysis of a group of piles submitted to an obliquely incident wave, $4 \mathrm{th}$ International Conference Numerical Methods Geomechanics, Edmonton, Balkema.

[20] CHAPEL F., GHAFFAR ZADEH M. (1983), Frequency independant impedances of soilstructures systems in horizontal and rocking modes, Earthquake Engineering and Structural Dynamics, vol. XI, pp. 523-540.

[21] CHAPEL F., TSAKALADIS C. (1985), Computation of the Green's functions of elastodynamics for a layered half space through a Hankel transform, Applications to foundation vibration and seismology, Numerical Methods in Geomechanics (Ed. Kawamoto, Ichikawa), Balkema, pp. 1311-1318.

[22] CHAPEL. F., CRÉPEL J.M. (1985), 3-D Analysis of a group of piles in a multilayered soil submitted to a seismic field, Comparisons with experiment, Numerical Methods in Geomechanics (Ed. Kawamoto, Ichikawa), Balkema, pp. 867. 874.

[23] CHAPEL F. (1986), Application de la méthode des équations intégrales à l'interaction solstructure linéaire sur un sol hétérogène. Actes Jer Colloque Français de Génie Parasismique, Association Française Génie Parasismique, vol. IV, pp. 37-48.

[24] CHOUVET D. (1983), Calcul élastoplastique d'interaction dynamique sol-structure, Thèse de Docteur Ingénieur, Ecole Centrale de Paris.

[25] CHU L.L., ASKAR A., CAKMAK A.S. (1981), An approximate method for soil-structure interaction for SH-waves, The Born approximation, Earthquake Engineering and Structural Dynamics, vol. IX, pp. 205-219.

[26] CRÉPEL J.M. (1983), Modélisation tridimensionnelle de l'interaction dynamique sol-structure par des éléments finis et infinis, Thèse de Docteur Ingénieur, École Centrale de Paris.

[27] DE SILVA N.S. (1984), Calculs d'interaction solstructures par la méthode des équations intégra. les, Applications aux fondations superficielles et enterrées et comparaison à des essais in situ, Thèse de Docteur Ingénieur, Êcole Centrale de Paris, le 20 septembre 1984.
[29] DAY S.M., FRAZIER G.A. (1979), Seismic response of hemispherical foundations, J. Engineering Mechanics Division ASCE, pp. 29-41.

[29] DRAVINSKI M., THAU S.A. (1976), Multiple diffractions of elastic shear waves by a rigid rectangular foundation embedded in an elastic halfspace, J. Applied Mechanics ASME, vol. XLIII, pp. 295-299.

[30] DRAVINSKI M., THAU S.A. (1976), Multiple diffractions of elastic waves by a rigid rectangular foundation: plane strain model, J. Applied Mechanics ASME, vol. XLIII, pp. 291-294.

[31] GAZETAS G.C. (1983), Analysis of machine foundations : state of the art, Soil dynamics and Earthquake Engineering, vol. Il-1, pp. 1-30.

[32] GUTIERREZ J.A., CHOPRA A.K. (1978), A substructure method for earthquake analysis of structures including structure-siol interaction, Earthquake Engineering and Structural Dynamics, vol. VI, pp. 51-69.

[33] HUJEUX J.C. (1985), Une loi de comportement pour le chargement cyclique des sols, Génie Parasismique (éd. Davidovici), Presses ENPC, pp. 287-302.

[34] JENNINGS P.C., BIELAK J. (1973), Dynamics of building-soil interaction, Bull. Seism. Soc. Am., vol. LXIII, pp. 9-48.

[35] IGUCHI M., LUCO J.E. (1981), Dynamic response of flexible rectangular foundations on an elastic halfspace, Earthquake Engineering and Structural Dynamics, vol. IX, pp. 239-249.

[36] IGUCHI M., LUCO J.E. (1982), Vibration of a flexible plate on a viscoelastic medium, J. Engineering Mechanics Division ASCE, vol. XXX, pp. XXX.

[37] KAUSEL E., ROESSET J.M., WAAS G. (1975), Dynamic analysis of footings on layered media, J. Engineering Mechanics Division ASCE, vol. CI, pp. 679-693.

[38] KAUSEL E., ROESSET J.M. (1975), Dynamic stiffness of circular foundations, J. Engineering Mechanics Division ASCE, vol. CI, pp. 771785.

[39] KENNETT B.L.N. (1983), Seismic wave propagation in stratified media, Cambridge University Press.

[40] KOBAYASHI S., NISHIMURA N. (1982), Transient stress analysis of tunnels and caverns of arbitrary shape due to travelling waves, in Developments in Boundary Elements, vol. II (Eds P.K. Banerjee and R.P. Shaw), Applied Science Publishers London.

[41] KOBORI T., MINAI R., SHINOZAKI Y. (1976), Vibration of a rigid circular disk on an elastic half. space subjected to plane waves, Theoretical and Applied Mechanics, University Tokyo Press, vol. XXI, pp. 109-119.

[42] KOBORI T., MINAI R., SHINOZAKI Y. (1976), Vibration of a rigid circular disk on an elastic halfspace subjected to plane waves (Part 2), Theoretical and Applied Mechanics, University Tokyo Press, vol. XXIV, pp. 153-167. 
[43] KUPRADZE V.D. (1963), Dynamical Problems in Elasticity, Progress in Solid Mechanics, North Holland.

[44] LUCO J.E., WETSMANN R.A. (1971), Dynamic response of circular footing, J. Applied Mechanics ASME, vol. XCVII, pp. 1381-1395.

[45] LUCO J.E., WETSMANN R.A. (1972), Dynamic response of a rigid footing bonded to an elastic half-space, J. Engineering Mechanics Division ASCE, vol, XXXIX, pp. 527-534.

[46] LUCO J.E. (1974), Impedance functions for a rigid foundation on a layered medium, Nuclear Engineering and Design, vol. XXXI, pp. 204-217.

[47] LUCO J.E., WONG H.L., TRIFUNAC M.D. (1975), A note on the dynamic response of rigid embedded foundations, Earthquake Engineering and Structural Dynamics, vol. IV, pp. 119-128.

[48] LUCO J.E. (1976), Vibrations of a rigid disk on a layered viscoelastic medium, Nuclear Engineering and Design, vol. XXXVI, pp. 325-340.

[49] LUCO J.E. (1976); Torsional response of structures for obliquely incident seismic waves, Earthquake Engineering and Structural Dynamics, vol. IV, pp. 207-219.

[50] LUCO J.E. (1976), Torsional response of structures for SH waves: the case of hemispherical foundations, Bull. Seism. Soc. Am., vol. LXVI-1, pp. 109-124.

[51] LUCO J.E., WONG H.L. (1977), Dynamic response of rectangular foundations for Rayleigh wave excitation, Proc. 6th World Conference Earthquake Engineering, New Delhi, India.

[52] LUCO J.E. (1980), Linear soil-structure interaction, Report UCRL-15272, Lawrence Livermore National Laboratory.

[53] LUCO J.E., APSEL R.J. (1982), On the Green's functions for layered media : part 1 and 2, Bull. Seism. Soc. Am., vol. LXXIIl-4, pp. 909-931.

[54] MILLER R.K. (1977), An approximate method of analysis of the transmission of elastic waves through a frictional boundary, J. Appl. Mechanics, ASME, vol. XXXVII, pp. 720-727.

[55] MILLER R.K. (1979), The effect of boundary friction on the propagation of elastic waves, Bull. Seism. Soc. Am., vol. LXVIII, pp. 987-998.

[56] MILLER R.K. (1979), Reflection, refraction, and absorption of elastic waves at a frictional interface: SH motion, J. Appl. Mechanics, ASME, vol. XLVI, pp. 625-630.

[57] MODARESSI H. (1986), Modélisation par éléments finis de la propagation des ondes en milieu poreux saturés, Application au génie parasismique, Thèse de Doctorat, École Centrale de Paris.

[58] NOGAMI T., NOVAK M. (1977), Resistance of soil to horizontally vibrating pile, J. Earthquake Engineering and Structural Dynamics, vol. V, pp. 249-261.

[59] NOGAMI T. (1985), Flexural responses of grouped piles under dynamic loading, J. Earthquake Engineering and Structural Dynamics, vol. XIII, pp. 321-336.
[60] NOVAK M., BEREDUGO Y.O. (1972), Vertical vibration of embedded footings, Soil Mechanics Foundation Division ASCE, vol, XCVIII, pp. 1291-1310.

[61] NOVAK M., NOGAMI T. (1977), Soil-pile interaction in horizontal vibration, J. Earthquake Engineering and Structural Dynamics, vol. V, pp. 263-282.

[62] NOVAK M., MOGAMI T., ABOUL ELLA F, (1978), Dynamic soil reaction for plane strain case, J. Engineering Mechanics Division ASCE, vol. CIV, pp. 953-959.

[63] NOVAK M., ABOUL ELLA F. (1978), Impedance function of piles in layered media, J. Engineering Mechanics Division ASCE, vol. CIV, pp. 643-661.

[64] OHTSUKI A., HARUMI K. (1983), Effect of topographies and subsurface inhomogeneities on seismic SV waves, Earthquake Engineering and Structural Dynamics, vol. XI, pp. 441-462.

[65] OHTSUKI A., YAMAHARA H., HARUMI K. (1984), Effect of topographies and subsurface inhomogeneities on seismic Rayleigh waves, Earthquake Engineering and Structural Dynamics, vol. XII, pp. 37-58.

[66] POSTEL M. (1985), Homogénéisation et réponse sismique de fondations sur pieux, Thèse de Docteur Ingénieur, École Centrale de Paris, le 5 juillet.

[67] RIGGS H.R., WAAS G. (1985), Influence of foundation flexibility on soil-structure interaction, Earthquake Engineering and Structural Dynamics, vol. XIII, pp. 597-616.

[68] SATAYOPAS B. (1983), Méthode analytique approchée de calcul de structures sur groupes de pieux dans un sol multicouche, Thèse de Docteur Ingénieur, École Centrale de Paris.

[69] SCANLAN R.H. (1976), Seismic wave effects on soil-structure interaction, Earthquake Engineering and Structural Dynamics, vol. IV, pp. 379 388.

[70] SEN R., DAVIES T.G., BANERJEE P.K. (1985), Dynamic analysis of piles and pile groups embedded in homogeneous soils, Earthquake Engineering and Structural Dynamics, vol. XIII, pp. 53-66.

[71] TRIFUNAC M.D. (1973), Scattering of plane SH-waves by a semi-cylindrical canyon, Earthquake Engineering and Structural Dynamics, vol. I, pp. 267-281.

[72] TSAI N.C., NIEHOFF D, SWATTA M., HADJIAN (1974), The use of frequency-independant soil-structure interaction parameters, Nuclear Engineering and Design, vol. XXXI, pp. 168183.

[73] TSAKALADIS C. (1985), Diffraction d'ondes sismiques sur les structures sur pieux et fonctions de Green du sol multicouche, Thèse de Docteur Ingénieur, École Centrale de Paris, le 5 juillet 1985.

[74] WASHIZU K. (1975), Variational methods in elasticity and plasticity. Pergamon Press, 2nd Edition. 
[75] WHITTAKER W.L., CHRISTIANO P. (1982), Response of a plate and elastic half-space to harmonic waves, Earthquake Engineering and Structural Dynamics, vol. X, pp. 255-266.

[76] WOLF J.P. (1976), Soil-structure interaction with separation of base from soil, Nuclear Engineering and Design, vol. XXXVIIl, pp. 463-473.

[77] WOLF J.P. (1977), Siesmic response due to travelling shear wave including soil-structure interaction with base-mat uplift. J. Earthquake Engineering and Structural Dynamics, vol. V, pp. 337. 364.

[78] WOLF J.P. (1979), Dynamic stiffness and seiismic input motion of a group of battered piles, Nuclear Engineering and Design, vol. LIV-3.

[79] WOLF J.P., ARX G.A. (1982), Horizontally travelling waves in a group of piles taking pile-soil. pile interaction into account, Earthquake Engineering and Structural Dynamics, vol. X, pp. 225 . 237.

[80] WOLF J.P., DARBRE G.R. (1983), Dynamic stiffness matrix of embedded and pile foundations by indirect boundary element methods, 7th SMIRT Conference, Chicago.

[81] WOLF J.P., DARBRE G.R. (1984), Dynamic stiffness matrix of soil by the boundary-element method : conceptual aspects, Earthquake Engineering and Structural Dynamics, vol. XII, pp. 385-400.
[82] WOLF J.P., DARBRE G.R. (1984), Dynamic stiffness matrix of soil by the boundary-element method: embedded foundations, Earthquake Engineering and Structural Dynamics, vol. XII, pp. $401-416$.

[83] WOLF J.P. (1985), Dynamic soil-structure interaction, Prentice Hall.

[84] WOLF J.P., OBERNHUBER P. (1985), Nonlinear soil-structure-interaction using dynamic stiffness or flexibility of soil in the time domain. Earthquake Engineering and Structural Dynamics, vol. XIII, pp. 195-212.

[85] WOLF J.P., OBERNHUBER P. (1985), Non. linear soil-structure-interaction using Green's function of soil in the time domain. Earthquake Engineering and Structural Dynamics, vol. XIII, pp. 195-212.

[86] WONG H.L., LUCO J.E. (1976), Dynamic response of rigid foundations of arbitrary shape. Earthquake Engineering and Structural Dynamics, vol. IV, pp. 579-587.

[87] WONG H.L., LUCO J.E. (1976), Dynamic res. ponse of rectangular foundations to obliquely incident seismic waves, Earthquake Engineering and Structural Dynamics, vol. VI, pp. 3-16.

[88] WONG H.L. (1982), Effect of surface topography on the diffraction of $P, S V$, and Rayleigh waves, Bull. Seism. Soc. Am., vol. LXXII, pp. 1167-1183. 